Ann. Sci. forest., 1968, 25 (2), 83-117.

\title{
VARIATIONS SAISONNIÈRES DES TENEURS EN EAU ET ÉLÉMENTS MINÉRAUX DES FEUILLES DE CHÊNE (QUERCUS PEDUNCULATA)
}

\author{
P. LEROY \\ avec la collaboration technique de Maryse Bitsch et G. Courrier \\ Station de Recherches sur les Sols forestiers et la Fertilisation, \\ Centre national de Recherches forestières, 54 - Nancy \\ Institut national de la Recherche agronomique
}

SOMMAIRE

Grâce à des mensurations, des pesées et des analyses chimiques, se sont dégagés quelques enseignements relatifs au diagnostic foliaire du chêne. L'objectif premier de cette étude était de mettre au point les règles d'échantillonnage foliaire adaptées à un programme de travail sur la nutrition et la production du chêne par comparaison de stations. Cet objectif a été, en quelque sorte, dépassé dans le sens où des indications intéressantes sont apportées à propos de la distribution de quelques éléments minéraux majeurs dans la masse foliaire du chêne. La méthode consistant à suivre simultanément la croissance des feuilles, les variations de teneurs en eau et en éléments minéraux s'avère ainsi très féconde.

\section{1. - INTRODUCTION ET GÉNÉRALITÉS}

Cette étude est le point de départ d'un programme de recherches sur la nutrition du chêne. Elle ne visait $a$ priori qu'à mettre au point les techniques d'échantillonnage foliaire du chêne. En fait, les premiers résultats dépassent ce simple aspect technique et apportent des enseignements dont la publication nous semble utile.

Précisons tout d'abord que nous avons pris le diagnostic foliaire comme méthode de travail privilégiée, pour aborder les problèmes de nutrition du chêne. Nous revien- 
drons sur ce point, mais remarquons au passage que les analyses de feuilles ne posent guère de difficultés au laboratoire; cette seule qualité leur confère un avantage appréciable sur les analyses de sol.

Il nous semble en outre nécessaire de commenter ici certains aspects de notre programme de travail sur le chêne, afin de placer dans leur contexte général les résultats partiels qui fournissent matière à cet article.

\section{1. - Pourquoi le chêne}

S'intéresser au chêne à l'époque des enrésinements massifs peut paraître paradoxal. Il importe donc de justifier ce choix en précisant que nous poursuivons un double objectif :

- déterminer en premier lieu les conditions de nutrition du chêne en fonction des types de sol et en liaison avec la production. De cette façon, nous pensons aider à la mise au point d'une sylviculture apte à fournir du bois de qualité aux moindres frais.

- A plus long terme, étalonner un instrument commode permettant de juger des potentialités nutritives d'une station en vue d'enrésinement. Le chêne étant une essence très répandue en France, la connaissance des corrélations entre nutrition du chêne et nutrition des résineux nous donnerait, en effet, le moyen de prévoir et même d'améliorer les possibilités de nutrition d'une plantation résineuse sur une station, par analyse foliaire des chênes préexistants.

Dans l'optique d'une forêt productive, cette double orientation s'adapte parfaitement aux préoccupations des forestiers qui cherchent à la fois à produire des feuillus de qualité et des résineux en quantité. Ce type de recherche sur le chêne contribue, par ailleurs, à apporter indirectement quelques connaissances à propos de la physiologie des arbres feuillus.

\section{2. - Diagnostic foliaire et études de nutrition des arbres forestiers}

\subsection{Remarques préliminaires}

La démarche naturelle pour étudier la nutrition minérale des végétaux consisterait à se pencher à la fois sur les propriétés des sols et sur les analyses foliaires. Malheureusement, les études de sol se prêtent mal aux interprétations. La fertilité du sol dépend en effet d'une quantité de facteurs physiques et chimiques dont la liste n'est jamais limitative. Et quand bien même on limiterait par un travail préliminaire les analyses à un nombre réduit de variables prépondérantes, se poseraient encore certaines difficultés expérimentales ou théoriques. On peut aussi penser avec de LEENHEER (1963) que les analyses de sol traduisent parfois davantage des réalités de laboratoire que des réalités de terrain. Dans ces conditions, on conçoit qu'elles n'ont de valeur explicative dans l'étude des problèmes de nutrition que pour des 
comparaisons de stations forestières à caractères tranchés, ce qui n'est pas le cas dans notre travail sur le chêne.

Il semble heureusement que les analyses foliaires posent au départ moins de difficultés théoriques et matérielles. Basées sur le concept que la plante elle-même est le meilleur indicateur de sa nutrition, elles ouvrent des perspectives intéressantes dans la mesure où elles intègrent l'ensemble des processus qui président à la nutrition minérale. Nous avons donc choisi le diagnostic foliaire comme méthode de travail préférentielle. Nous n'en négligerons pas pour autant les analyses classiques du sol qui restent indispensables à la fois pour définir certaines caractéristiques de station et guider les éventuelles opérations d'amélioration.

\subsection{Eléments bibliographiques}

Les analyses foliaires bénéficient déjà d'un vaste champ d'application dans le domaine forestier. Cependant, pour des raisons d'urgence économique, la majorité des chercheurs les ont essentiellement utilisées dans des études de nutrition sur résineux. Les incursions dans le « feuillu » sont bien rares, mis à part quelques résultats sur peuplier (GuILIMONDI, 1960, Van der MEIDEN, 1965) ou sur eucalyptus.

Ainsi la base de départ de notre travail sur le chêne se trouvait-elle assez réduite. Sur le plan des méthodes, il nous restait quand même l'expérience acquise avec les résineux.

Si les feuillus n'ont guère été pris en considération, il faut cependant reconnaître que les quelques travaux publiés à leur sujet présentent un certain intérêt. Ils n'abordent cependant bien souvent qu'un aspect très limité des problèmes. Ainsi FIEDLER et HoHNE (1965) ont pu dresser une revue des teneurs en macréoléments pour divers feuillus. Ces données ne sont malheureusement liées ni à la production, ni à la station et les conditions d'échantillonnage ne sont pas précisées. Dans l'état actuel de nos connaissances, ces résultats permettent pourtant des comparaisons valables.

Par ailleurs, le travail de GuHA et Mrtchell (1965) mérite d'être spécialement mentionné. Sur feuilles d'érable et hêtre, ces auteurs ont en effet étudié avec soin les variations saisonnières de teneurs en un certain nombre d'éléments minéraux, ce qui constitue un des rares essais de ce genre pour les feuillus. Nous n'avons pas hésité à nous inspirer de quelques-unes de leurs méthodes.

\section{3. - Objectifs fixes à l'étude présente}

Les quelques éléments bibliographiques que nous avons pu recueillir, nous ont aidé à préciser les objectifs de notre travail sur le chêne. Ainsi en abordant des comparaisons de stations et de productions, il nous fallait simultanément mettre au point les techniques d'échantillonnage foliaire du chêne et nous procurer quelques informations susceptibles de nous guider dans les interprétations d'analyse. Notre programme de recherches s'est donc naturellement découpé en deux tranches :

- étude théorique du diagnostic foliaire sur une station de chênes à proximité de Nancy (forêt de Champenoux); 
- comparaison de douze stations de chênes réparties dans trois forêts à l'ouest et au sud du Bassin parisien.

Nous comptons sur les enseignements acquis à partir de ces deux tranches pour atteindre les objectifs généraux définis au paragraphe 1.1.

Les résultats partiels que nous commentons ici ont trait à la station de Champenoux. Ils concernent les prélèvements réalisés courant 1966 et même 1967 pour certains caractères. Le but premier de ces campagnes était d'étudier les variations saisonnières de teneurs en éléments minéraux des feuilles de chêne afin d'établir les courbes de variations en fonction de l'âge des feuilles et aussi en fonction de la hauteur d'échantillonnage dans la couronne. A partir de ces résultats, il est en effet possible de déterminer :

- les dates et positions d'échantillonnage les plus favorables pour l'étude de la nutrition minérale ;

- le nombre d'arbres nécessaires pour obtenir une précision suffisante.

Les résultats présentés sont partiels dans la mesure où il faudra répéter les mêmes opérations pendant quelques années pour tenir compte de l'influence du climat (Miller, 1967). Cependant le forme des courbes obtentues sur une année de végétation semble être une caractéristique physiologique peu modifiée par les aléas climatiques.

Nous avons, en outre, profité de nos prélèvements foliaires pour chiffrer avec quelque précision les variations de poids sec, de taille et de teneur en eau des feuilles. De cette façon, nous pensions réunir un certain nombre d'idées exploitables par les physiologistes dans leurs études sur les mécanismes de distribution des éléments minéraux.

Ainsi, en définitive, le présent article fournira-t-il deux séries de résultats : l'une rapportant des résultats de mensurations et de pesées donc des données physiques, la seconde rapportant des résultats d'analyses chimiques.

\section{2. - MATÉRIEL ET MÉTHODES}

\section{1. - Caractíristiques de la station de Champenoux (M.-et-M.) (15 km notd de Nancy)}

Nous avons choisi en forêt domaniale de Champenoux un peuplement régulier de chènes pédonculés (Quercus pedunculata) âgés de 20 ans avec quelques charmes (Carpinus betulus) en sous-étage. L'indice de production exprimé par l'accroissement moyen annuel maximum s'élève à $8,1 \mathrm{~m}^{3}$, Cet indice a été calculé par J. BouchoN, Station de Sylviculture du C.N.R.F. Les prélèvements ont été effectués sur 10 arbres dominants répartis sur 5 ares et présentant les mensurations moyennes des dominants de la station, hauteur moyenne de $11,8 \mathrm{~m}$, circonférence moyenne de $45 \mathrm{~cm}$.

Le sol de type brun, mésotrophe à pseudogley est assis sur argiles du sinémurien supérieur recouvert d'un léger placage de limons; enracinement sur un mètre environ. Le sol partiellement désaturé est relativement pauvre en $\mathrm{P}_{5} \mathrm{O}_{5}$. On peut relever dans la strate herbacée discontinue la présence de Molinia coeruléa, Agrostis canina, Deschampsia coespitosa mais également de la ronce (Rubus).

\section{2. - MÉTHODE D'ÉCHANTILLONNAGE:}

Les prélèvements ont lieu à intervalles égaux au cours de la saison de végétation. Ils commencent à la fin du $\propto$ débourrage $»$ pour se terminer avec la sénescence. 
Les échantillons sont récoltés le même jour et par temps clair à trois hauteurs différentes de la couronne : tiers supérieur, médian et inférieur $(\mathrm{H}, \mathrm{M}, \mathrm{B})$. Pour un échantillon, les feuilles proviennent de sept à huit portions moyennes de branches prises tout autour de l'arbre, de façon à éliminer les effets d'orientation.

Le poids frais moyen d'un échantillon est d'environ 50 à $70 \mathrm{~g}$; ce poids, suffisant pour donner un échantillon représentatif, est assez modeste pour éviter une défoliation excessive des arbres retenus. Les feuilles, empaquetées sur le terrain dans des sacs de plastique étanches et purgés d'air au maximum, sont placées en chambre froide trois heures aprés la récolte. Elles n'en sortent le lendemain que pour les diverses mensurations et le séchage en étuve à $65^{\circ}$ pendant 24 heures ; on peut considèrer que la perte de poids par dessiccation provient uniquement de l'élimination de l'eau (Demolon, 1956). Aprés le séchage, les échantillons sont pesés, broyés finement et stockés dans des boites de plastique étanches.

Au cours des mensurations, les feuilles parasitées sont rejetées; le taux d'élimination n'a jamais dépassé $10 \%$ du poids frais en 1966, année oú la tordeuse du chêne (Tortrix viridana) était assez abondante. Les attaques de cette chenille étaient concentrées sur le haut des cimes ; fin juin, la nymphose a fait disparaitre les dégâts. Nous avons vérifié que le parasitisme n'affectait pas significativement nos résultats d'analyse chimique.

\section{3. - MENSURATIONS PRÉALABLES AUX ANALYSES CHIMLULS}

Avant de passer à l'étuve, chaque échantillon frais fait l'objet d'une série d'observations : comptages, pesées, mesures des dimensions des limbes. A la sortie de l'étuve, les échantillons sont immédiatement pesés de façon à avoir une valeur aussi exacte que possible des poids secs.

Ces diverses observations permettront d'interpréter les variations de teneurs en éléments minéraux en fonction de données relatives au développement végétatif des feuilles.

\section{4. - Méthodes d'ANALYSes ChIMQQues}

Pour démarrer notre programme, nous n'avons retenu que cinq éléments majeurs : N, P, K. $\mathrm{Ca}, \mathrm{Mg}$. Nous pensons étendre éventuellement cette gamme à la lumière des enseignements apportés par nos études de stations.

Les feuilles ne subissent aucun nettoyage ou lavage avant d'être traitées. D'aprẻs GuHA et MiTCHELL (1966), les pollutions par les poussières atmosphériques n'affectent en rien les dosages des macroéléments. Il serait indispensable de revoir cette question si nous devions aborder le domaine des oligo-éléments.

La minéralisation des prises d'essai se réalise par attaque à l'acide sulfurique et l'eau oxygénée. Pour chacun des éléments, nous suivons les méthodes suivantes :

- Azote : dosage par distillation au micro-kjeldahl Parnas Wagner et titration avec $\mathrm{H}_{2} \mathrm{SO}_{4}$. Erreur au seuil de $5 \%: 2 \%$

- Phosphore : dosage par colorimétrie avec solution molybdique et chlorure stanneux. Erreur au seuil de $5 \%: 0 \%$.

- Potassium, calcium : dosage au photomètre de flamme en solution neutre.

$\mathrm{K}$, erreur au seuil de $5 \%: 1 \%$

Ca, erreur au seuil de $5 \%: 2 \%$.

- Magnésium : dosage par colorimétrie au jaune de titane.

Erreur au seuil de $5 \%: 10 \%$.

\section{3. - INFORMATIONS FOURNIES PAR LES MENSURATIONS PRÉALABLES}

Dans un premier temps, nous passerons en revue les trois types d'observations réalisées, pour essayer ensuite de tirer une conclusion globale. 


\section{1. - Mesures des teneurs en eau des feuilles}

\subsection{Généralités}

On peut constater de nombreuses divergences parmi les auteurs à propos de la base sur laquelle il faut présenter les résultats des mesures de teneur en eau. En effet, le poids frais, comme le poids sec sont sujets à des variations journalières ; il est toutefois évident que le poids sec, relativement indépendant du facteur " eau » est sensiblement plus constant sur une journée que le poids frais. Nous avons ainsi retenu le poids sec comme référence dans l'expression des teneurs en eau. Dorénavant, nous noterons le $\%$ d'eau par rapport au poids sec par le symbole $\% \mathrm{H}_{2} \mathrm{O}$.

A l'avenir, nous pensons améliorer la présentation et l'intérêt des données concernant les teneurs en eau en introduisant la notion de turgescence relative (de Parcevaux, 1963).

En ce qui concerne les premiers résultats acquis, il nous faut noter une très grande variabilité des $\% \mathrm{H}_{2} \mathrm{O}$ en fonction de l'époque et de la position d'échantillonnage (tableau I). Ainsi en 1966, la fourchette s'étendait de 120 à $200 \%$ et en 1967 de 125 à $330 \%$. La grande différence entre les valeurs de départ 1966 et 1967 peut être attribuée en partie au fait que les prélèvements ont débuté en 1967 plus vite après le débourrement, donc sur feuilles plus jeunes qu'en 1966.

Par ailleurs, les dix arbres n'étant pas, par impossibilité matérielle, échantillonné aux mêmes heures, il est difficile de prendre en compte ici les $\% \mathrm{H}_{2} \mathrm{O}$ individuels pour une date déterminée. $\mathrm{Ces} \% \mathrm{H}_{2} \mathrm{O}$ varient en effet très rapidement au cours de la journée. Dans le dessein de préciser ces variations, des prélèvements sur quelques arbres ont été répétés au même niveau un 7 août à 8 heures et à 15 heures : la diminution moyenne observée du $\% \mathrm{H}_{2} \mathrm{O}$ est de l'ordre de 12 , ce qui correspond sensiblement aux chiffres cités par de PARCEVAux à propos de la transpiration végétale.

Dans le même ordre d'idée, deux prélèvements différents ont été réalisés à la même heure sur quelques arbres. Les erreurs d'appréciation sur le $\%, \mathrm{H}_{2} \mathrm{O}$ dues à l'échantillonnage ont pu ainsi être évaluées à $5 \%$.

En définitive, notre méthode d'échantillonnage ne permet pas de suivre de près le comportement individuel des arbres vis-à-vis de l'eau. Seuls, les $\% \mathrm{H}_{2} \mathrm{O}$ moyens obtenus à partir des 10 arbres ont une signification réelle. Il apparait cependant avec certitude que les feuilles de certains arbres ont une propension à être plus riches en eau que d'autres ; ainsi, par exemple, 2 individus voisins, grimpés à dessein sensiblement toujours à la même heure, voient à chaque époque leur $\% \mathrm{H}_{2} \mathrm{O}$ différer grandement et dans le même sens. La comparaison des moyennes de leurs teneurs en eau pour 1966 et 1967 illustre bien cet antagonisme :

$$
\% \mathrm{H}_{2} \mathrm{O}-1966
$$

Arbre 7

Arbre 4
171.8 143.9

$$
\% \mathrm{H}_{2} \mathrm{O}-1967
$$

194.4

Nous reprendrons plus bas ces considérations sur les comportements individuels vis-à-vis de l'eau. 


\subsection{Résultats}

La plus petite différence significative pour les données du tableau 1 étant de l'ordre de $10 \%$, il nous est facile de suivre les variations dans le $\% \mathrm{H}_{2} \mathrm{O}$, à la fois en fonction de l'époque et de la position d'échantillonnage.

La décroissance du $\% \mathrm{H}_{2} \mathrm{O}$ au cours de la saison de végétation, déjà citée par KozlowsKı (1966) se trouve confirmée dans le cas du chêne. Elle est très rapide en début de saison; il semble toutefois possible d'admettre que le $\% \mathrm{H}_{2} \mathrm{O}$ varie relativement peu à partir de début août, exceptée une tendance à la décroissance en octobre.

Les écarts importants entre les $\% \mathrm{H}_{2} \mathrm{O}$ de départ en 1966 et 1967 peuvent s'expliquer par le fait que le débourrage a été plus précoce en 1966 qu'en 1967, par suite probablement des températures plus fraîches en mai 1967 qu'en mai 1966 (1). Ainsi, fin mai juin 1967, les feuilles étaient bien jeunes.

En poussant les comparaisons entre 1966 et 1967, il apparaît que les $\% \mathrm{H}_{2} \mathrm{O}$ atteignent des niveaux sensiblement voisins à partir de fin juillet. II semble même que la sécheresse de juillet et août 1967 ait marqué la nutrition en eau sur la station (comparaison des $\% \mathrm{H}_{2} \mathrm{O}$ d'août sur les deux années). Nous pouvons ainsi retenir que les écarts provoqués par des dates de débourrage différentes s'atténuent au cours de la saison de végétation.

En définitive, l'ensemble des faits précédents paraît indiquer que des prélèvements foliaires réalisés de façon homogène, au mois d'août de la même année, conviendraient pour des comparaisons de teneurs en eau des feuilles sur diverses stations.

En nous intéressant maintenant à l'effet de la position d'échantillonnage sur le $\% \mathrm{H}_{2} \mathrm{O}$, nous pouvons constater que les feuilles du bas sont plus riches en eau que les feuilles du haut (tableau 1). Cette tendance est d'ailleurs beaucoup plus nette en 1967 qu'en 1966. Nous avons pu vérifier cette action de la position en prélevant des gourmands à chaque date et en milieu de journée pour nous placer dans les conditions les plus démonstratives : dès la fin juin, les résultats figurant au tableau 1 confirment la tendance à la richesse relative en eau des feuilles d'ombre par rapport à celles de lumière. PISEK et Winckler (1956) cités par de PARCEVAUX avaient rapporté des conclusions de même nature pour le hêtre, en indiquant que le $\% \mathrm{H}_{2} \mathrm{O}$ optimum était de $170,3 \%$ pour les feuilles d'ombre et de $138,1 \%$ pour les feuilles de lumière.

Ces mêmes auteurs citaient d'ailleurs les chiffres suivants pour les $\% \mathrm{H}_{2} \mathrm{O}$ des feuilles de chêne en milieu de saison :

optimum : $156 \%$

début fermeture des stomates : $143 \%$

fin fermeture des stomates : $125 \%$

flétrissement : $84 \%$.

(1) Données climatologiques fournies par la Station météorologique de Champenoux, C.N.R.F., Sylviculture. 
Dans la mesure où celà est possible, nous pouvons admettre, d'après ces données, que nos chênes de Champenoux souffrent les jours d'été d'un léger déficit de saturation en eau. Nous avons d'ailleurs vérifié que le 7 août 1967 , le $\% \mathrm{H}_{2} \mathrm{O}$ à la turgescence des feuilles du bas pour nos dix arbres était de l'ordre de $160 \%$, ce qui donne un déficit de saturation d'environ $8 \%$.

\section{TABLEAU 1}

Teneur en eau des feuilles exprimée en fonction du poids sec $\left(\% \mathrm{H}_{2} \mathrm{O}\right)$ Variations suivant l'époque et la position d'échantillonnage Moyenne des observations sur 10 arbres

$H, M, B$ : Tiers supérieur, médiant et inférieur du Houppier

\begin{tabular}{|c|c|c|c|c|c|c|}
\hline 1966 & $1^{\text {ef }}$ juin & 29 juin & 26 juil. & 24 août & 20 sept. & 18 oct. \\
\hline Moyenne des 3 positions, & 195,6 & 130,4 & 139,9 & 146,4 & 142,7 & 129,3 \\
\hline$H$ & 191,6 & 132,3 & 136,3 & 136,1 & 135,2 & 1247 \\
\hline$M$ & 191,7 & 123,4 & 130,1 & 137,7 & 139,4 & 127,9 \\
\hline B & 203,4 & 135,2 & 153,3 & 165,1 & 153,6 & 135,5 \\
\hline Gourmands & 158,0 & 234,0 & 183,0 & 195,6 & 169,3 & \\
\hline
\end{tabular}

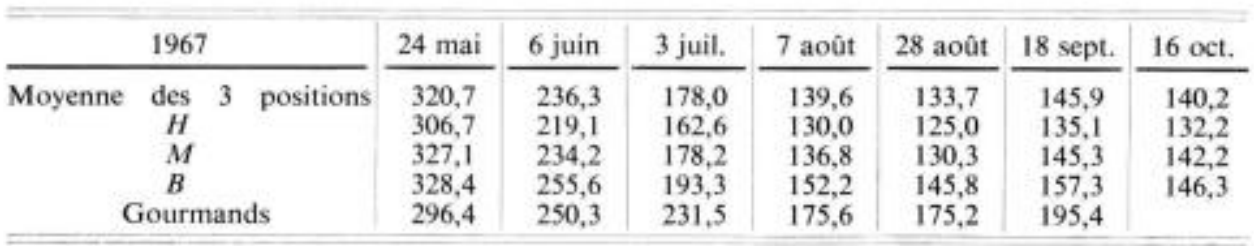

\section{TABLEAU 2}

Poids sec de 1000 feuilles en grammes

Variations 1966 en fonction de l'époque et de la position d'échantillonnage Moyenne des mesures sur 10 arbres

\begin{tabular}{c|cc|c}
\hline \hline 1966 & 24 août & 20 sept. & 18 oct. \\
\cline { 3 - 3 } Moyenne des 3 positions & 215,7 & 221,6 & 281,0 \\
$H$ & 243,4 & 238,1 & 363,5 \\
$M$ & 219,3 & 233,6 & 270,0 \\
$B$ & 184,6 & 193,2 & 209,6 \\
\hline \hline
\end{tabular}

\subsection{Conclusion partielle}

Notre méthode d'échantillonnage, en étalant les prélẻvements sur l'ensemble d'une journée ne nous fournit que peu d'informations sur le comportement individuel des arbres vis à vis de l'eau. Elle permettra de suivre les variations dans les $\% \mathrm{H}_{2} \mathrm{O}$ moyens dans le temps et dans l'espace.

Pour obtenir le maximum de renseignements, il semble opportun de prendre les précautions suivantes :

- échantillonner en août, à l'époque du minimum de variations du $\% \mathrm{H}_{2} \mathrm{O}$; 
- opérer le matin et par temps couvert si possible de façon à minimiser les variations journalières ;

- prélever à mi-hauteur du houppier, oủ le $\% \mathrm{H}_{2} \mathrm{O}$ semble représentatif de l'ensemble du feuillage ; à $10 \%$.

- récolter sur dix arbres pour mettre en évidence les différences supérieures

En respectant ces directives, il est possible de comparer les $\% \mathrm{H}_{2} \mathrm{O}$ de diverses stations. Nous avons pu vérifier que ce type de comparaisons ne manquait pas d'intérêt (résultats en cours de publication).

\section{2. - Mesures sur les poids secs : poids de 1000 feuilles sèches}

\subsection{Variations moyennes pour les dix arbres}

La figure 1 et le tableau 2 nous indiquent les tendances générales des variations de poids sec au cours de la saison de végétation. L'indice de poids sec retenu est le poids sec de 1000 feuilles ou Ps. Nous notons une augmentation continue du Ps

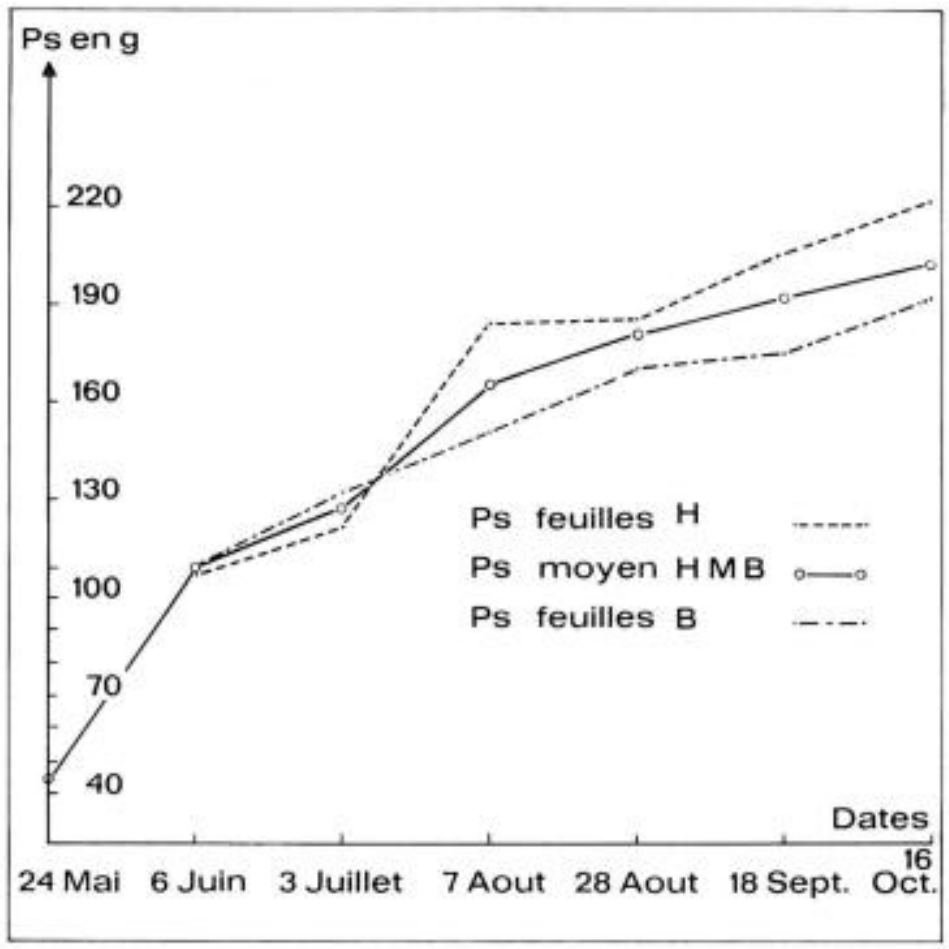

FiG. 1. - 1967 - Variations du poids see de 1000 feuilles (Ps) suivant l'époque et la position d'échantillonnage 
de juin à octobre ; en 1967, par exemple, le Ps moyen des trois positions passe de 46 à $204 \mathrm{~g}$, plus de la moitié de ce gain étant acquis en fin juin. La phase de croissance rapide de $\mathrm{Ps}$ correspond d'ailleurs à la période où les $\% \mathrm{H}_{2} \mathrm{O}$ sont les plus forts. Il est probable qu'à cette période les déficits de saturation en eau sont trop faibles pour limiter sensiblement l'assimilation par fermeture des stomates.

La comparaison des allures des courbes de variation de Ps pour le haut et le bas $d u$ houppier est surprenante. Ainsi, pour des valeurs de Ps voisines en début de saison, la croissance du Ps est continue pour les feuilles du bas tandis qu'elle semble marquer un palier en août pour les feuilles du haut. Ce palier n'est, à notre avis, que la manifestation d'une deuxième pousse ou pousse d'été sur le Ps ; sa signification se trouve donc réduite au fait que les échantillons du haut contiennent en août des feuilles en moyenne plus jeunes que ceux du bas. Nous avons d'ailleurs pû constater, grâce à des observations morphologiques, que cette pousse d'été n'affecte que les parties de l'arbre les plus éclairées, donc globalement les plus hautes. Pour l'ensemble de la saison, le taux de croissance des feuilles du haut est significativement plus élevé que pour le bas. Cette opposition de comportement apparaît aussi bien en 1966 qu'en 1967 (fig. 1, tabl. 2). Il est donc vraisemblable que les feuilles du haut se caractérisent par une activité physiologique plus intense et de plus longue durée que celles du bas. Afin d'illustrer cette opposition, nous avons calculé les taux relatifs de croissance obtenus en formant le rapport :

$$
\text { T.R.C. }=\frac{\text { Gain de poids pour une période donnée }}{\text { Poids moyen au cours de la même période }} \times 100
$$

Cet indice, utilisé par WARIENG (1966) est intéressant dans la mesure où il donne une première idée des possibilités d'assimilation du chêne. La lecture du tableau 3 montre bien la décroissance générale du T.R.C. au cours de la saison de végétation et confirme nos remarques à propos des différences d'activités physiologiques entre les feuilles du haut et celles du bas. L'inversion d'août entre haut et bas doit correspondre au développement de la deuxième pousse.

En calculant, par ailleurs, le taux relatif de croissance global de mai à octobre 1967, pour l'ensemble de la couronne, nous trouvons une valeur de $0.011 \mathrm{~g} / \mathrm{g} / \mathrm{jour}$, chiffre qui correspond sensiblement à ceux de W.F. POLLARD pour le Pin sylvestre (Pollard, cité par Warieng, 1966).

TABLEAU 3

Taux relatif de croissance 1967

Variations en fonction de l'époque et de la position d'échantillonnage

\begin{tabular}{|c|c|c|c|c|c|}
\hline 1967 & mai-juin & juillet-août & août & sept-oct. & $\begin{array}{l}\text { Global } \\
\text { mai-oct. }\end{array}$ \\
\hline Moyenne des 3 positions & $\begin{array}{l}81,5 \\
89,8 \\
76,1\end{array}$ & $\begin{array}{l}20,2 \\
24,8 \\
16,7\end{array}$ & $\begin{array}{c}8,7 \\
0 \\
12,6\end{array}$ & $\begin{array}{l}6,0 \\
9,3 \\
6,3\end{array}$ & $\begin{array}{l}124,6 \\
136,3 \\
119.6\end{array}$ \\
\hline
\end{tabular}


En définitive, retenons surtout que les feuilles du haut, donc de lumière, fabriquent plus de matière sèche que celles du bas : les Ps du bas en 1966 et 1967 sont inférieurs aux Ps du haut. Ce résultat est en accord avec les connaissances acquises sur l'assimilation des feuilles d'ombre et de lumière.

Sur un tout autre plan, il n'est pas inintéressant de lier maintenant les variations du Ps et du $\% \mathrm{H}_{2} \mathrm{O}$. Globalement, le Ps croît alors que le $\% \mathrm{H}_{2} \mathrm{O}$ décroît au cours de la saison de végétation, exception faite des mois de septembre et octobre 1967 où le déficit de saturation en eau tend à s'atténuer après la sécheresse estivale (tableau 1).

Cette allure des phénomènes, déjà citée par KozLowskı (1966), peut essentiellement être attribuée à l'épaississement des parois cellulaires au cours de la saison de végétation. Toutefois, la différence entre les Ps moyens d'octobre 1966-1967 (281 contre 204) tend à montrer que la sécheresse de 67 a eu un effet dépressif sur la croissance en Ps. Cette dernière dépendrait donc, pour une part, des possibilités d'alimentation en eau.

\subsection{Variations individuelles de Ps}

Par opposition avec les $\% \mathrm{H}_{2} \mathrm{O}$, les $\mathrm{Ps}$ de chacun des 10 arbres sont des chiffres parfaitement comparables entre eux ; en effet, le $\% \mathrm{H}_{2} \mathrm{O}$, facteur variable sur quelques heures étant éliminé, il nous est possible d'admettre que les variations de Ps sont négligeables entre le début et la fin d'une récolte.

Les classements 1966 et 1967 des dix arbres, en fonction du Ps de fin de saison, sont identiques; les variations de rang d'un arbre, d'une année à l'autre, sont de zéro ou une unité. Les plus petits Ps, moyenne des trois positions, sont respectivement de 142 et $104 \mathrm{~g}$ en septembre 1966 et septembre 1967. Les plus grands sont de 366 et $315 \mathrm{~g}$, aux mêmes époques. Certains arbres fabriquent donc, indubitablement, des feuilles plus lourdes que d'autres. Ces variations individuelles, à fourchette étendue, peuvent être liées à de nombreux facteurs d'ordre génétique ou écologique. II est regrettable, sur ce plan, que nous ne puissions établir une corrélation globale précise entre les $\% \mathrm{H}_{2} \mathrm{O}$ et les $\mathrm{Ps}$.

Nous avons cependant cherché à lier les Ps de fin de saison 1966 et 1967 aux $\% \mathrm{H}_{2} \mathrm{O}$ moyens des deux années, pour chacun des 10 arbres. Aucune liaison significative n'apparait. Si cette liaison entre Ps de fin de saison et $\% \mathbf{H}_{2} \mathrm{O}$ moyens de la saison existe, elle est faible et ne pourrait être mise en évidence qu'avec des mesures de pourcentages d'eau plus élaborées.

Il n'apparait pas non plus de corrélation entre les Ps 1966 et 1967 et les dimensions, hauteur ou diamètre, des arbres. Là encore, bien d'autres facteurs que le poids sec de 1000 feuilles peuvent intervenir ; les volumes et dispositions des houppiers ont, à eux seuls, plus d'importance que le simple Ps. Nous envisageons cependant de prélever des carottes pour vérifier l'absence de liaison entre le Ps et les largeurs des derniers cernes. Dans le même ordre d'idée, nous réaliserons une étude de la forme et du volume des houppiers. 


\subsection{Conclusion partielle}

L'ensemble des résultats concernant les poids secs de 1000 feuilles nous permet de penser que :

- les feuilles du haut, donc feuilles de lumière, ont une activité physiologique plus intense que les feuilles d'ombre. Cette tendance se traduit par des Ps plus élevés dans le haut que dans le bas des houppiers. végétation.

- plus de la moitié de la croissance en Ps a lieu dans les deux premiers mois de

- les classements des arbres en fonction du Ps de fin de saison, sont permanents mais ne semblent liés, ni aux $\% \mathrm{H}_{2} \mathrm{O}$, ni aux dimensions individuelles des arbres.

- un échantillonnage sur dix arbres, et pour la position médiane, donne la moyenne de Ps à une époque quelconque avec une précision de 20 à $30 \%$. Cette relative imprécision n'exclut pourtant pas de fructueuses comparaisons « interstations ").

\section{3. - Dimensions des feuilles}

\subsection{Variations moyennes pour les dix arbres}

Comme on pouvait s'y attendre, il apparait, au tableau 4, que la croissance en dimensions est continue du début à la fin de la saison de végétation, compte tenu des aléas provoqués par la deuxième pousse sur les feuilles du haut essentiellement. Elles passent de $6,1 \times 3,4 \mathrm{~cm}$ en fin mai 1967 à $9,0 \times 5,4 \mathrm{~cm}$ en octobre 1967. En ce qui concerne les dimensions, l'effet de la deuxième pousse se manifeste début juillet, alors que pour les Ps, cet effet n'est vraiment marqué qu'en août par un palier pour les feuilles du haut.

Nous relevons également que les feuilles du haut, donc de lumière, sont régulièrement significativement plus petites que celles du bas. Cette tendance des feuilles d'ombre à prendre de l'extension est bien confirmée par les dimensions des gourmands qui sont, en septembre 1967 de $7,2 \times 12,3 \mathrm{~cm}$. Par ailleurs, si les poids secs quintuplent entre mai et octobre, nous voyons les dimensions augmenter dans une bien moins grande proportion au cours de la même période. En fait, au début de nos prélèvements, les feuilles avaient déjà atteint des dimensions respectables alors que la croissance en Ps ne faisait que commencer. Tout se passe comme si les feuilles faisaient de la surface avant de prendre de "l 'épaisseur et de la densité ». Remarquons au passage que les dimensions, contre toute attente, augmentent encore entre septembre et octobre. Cette observation semble fondée : le grand nombre de mesures par date (1 000 et 1500 ) d'une part, l'absence de chute importante de feuilles avant le dernier prélèvement, d'autre part, écartent l'hypothèse d'un biais d'échantillonnage.

Notons enfin qu'entre 1966 et 1967, apparaissent une fois de plus des différences certainement imputables à la sécheresse relative de 1967. Ainsi, les dimensions atteignaient $6,6 \times 10,3 \mathrm{~cm}$ en octobre 1966 contre $5,4 \times 9,0 \mathrm{~cm}$ en octobre 1967 . 
TABLEAU 4

Longueur et largeur des feuilles en $\mathrm{cm}$

Variations en fonction de I'époque et de la position d'échantillonnage 1967

\begin{tabular}{|c|c|c|c|c|c|c|}
\hline & & $24 \mathrm{mai}$ & 6 juin & 3 juil. & 18 sept. & 16 oct. \\
\hline 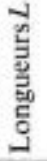 & $\begin{array}{c}\text { Moyenne } H M B \\
H \\
B \\
\text { Gourmands }\end{array}$ & $\begin{array}{l}6,09 \\
5,63 \\
6,41 \\
7,09\end{array}$ & $\begin{array}{l}8,49 \\
7,77 \\
8,98 \\
7,99\end{array}$ & $\begin{array}{r}8,07 \\
7,03 \\
9,03 \\
10,19\end{array}$ & $\begin{array}{r}8,42 \\
8,10 \\
8,95 \\
12,30\end{array}$ & $\begin{array}{l}9,01 \\
8,70 \\
9,40\end{array}$ \\
\hline 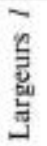 & $\begin{array}{c}\text { Moyenne } H M B \\
H \\
B \\
\text { Gourmands }\end{array}$ & $\begin{array}{l}3,41 \\
3,15 \\
3,50 \\
3,89\end{array}$ & $\begin{array}{l}5,03 \\
4,62 \\
5,33 \\
4,48\end{array}$ & $\begin{array}{l}4,60 \\
4,02 \\
5,12 \\
5,82\end{array}$ & $\begin{array}{l}5,00 \\
4,69 \\
5,43 \\
7,20\end{array}$ & $\begin{array}{l}5,40 \\
5,09 \\
5,60\end{array}$ \\
\hline
\end{tabular}

\subsection{Variations individuelles}

Nous avons pû suivre, en 67 , le développement en dimensions des feuilles, pour chacun des 10 arbres. Les différences individuelles sont importantes : en septembre, la fourchette allait de $3,8 \times 6,3 \mathrm{~cm}$ à $6 \times 10,1 \mathrm{~cm}$. Les classements en fonction des dimensions et des Ps sont très voisins. Quelle que soit la date, les corrélations Ps-dimensions sont très largement significatives. Par exemple, en 1967 :

$$
\begin{array}{lll}
\text { Ps - longueurs } & r=0,91 & \alpha<0,01 \% \\
\text { Ps - largeurs } & r=0,80 & \alpha<0,01 \%
\end{array}
$$

\section{4. - Mesures préalables : essai de synthèse}

Un certain nombre de nos premières conclusions seront affinées dans l'avenir, à l'aide de mensurations complémentaires. Toutefois, nous pouvons dès maintenant tirer deux séries d'enseignements, les uns relatifs à la description de la croissance des feuilles, les autres ayant trait aux problèmes d'échantillonnage foliaire. Soulignons, en outre, que les indications obtenues grâce à ces mesures préalables seront de précieux auxiliaires dans l'interprétation des analyses chimiques.

\subsection{Croissance des feuilles}

La figure 2 illustre l'ensemble de nos observations. Le pourcentage d'eau des feuilles décroit fortement du début à la fin de la saison de végétation ; le maximum de variation se place entre mai et juillet. Le poids sec de 1000 feuilles au contraire quintuple de mai à octobre, le gain le plus rapide se situant au début de cette période. Les dimensions des feuilles quant à elles augmentent de mai à octobre, l'époque de développement explosif des dimensions, juste après de débourrage n'a pu être suivie entièrement dans notre travail. 
L'accroissement du poids sec des feuilles s'accompagne ainsi d'une diminution dans le pourcentage d'eau de constitution. La croissance en poids sec se fait en outre davantage par " épaississement $)$ des feuilles que par augmentation des dimensions. L'ensemble de ces constatations se traduit par un taux de croissance relatif de l'ordre de $0,011 \mathrm{~g} / \mathrm{g} /$ jour, chiffre qui correspond à celui du pin sylvestre.

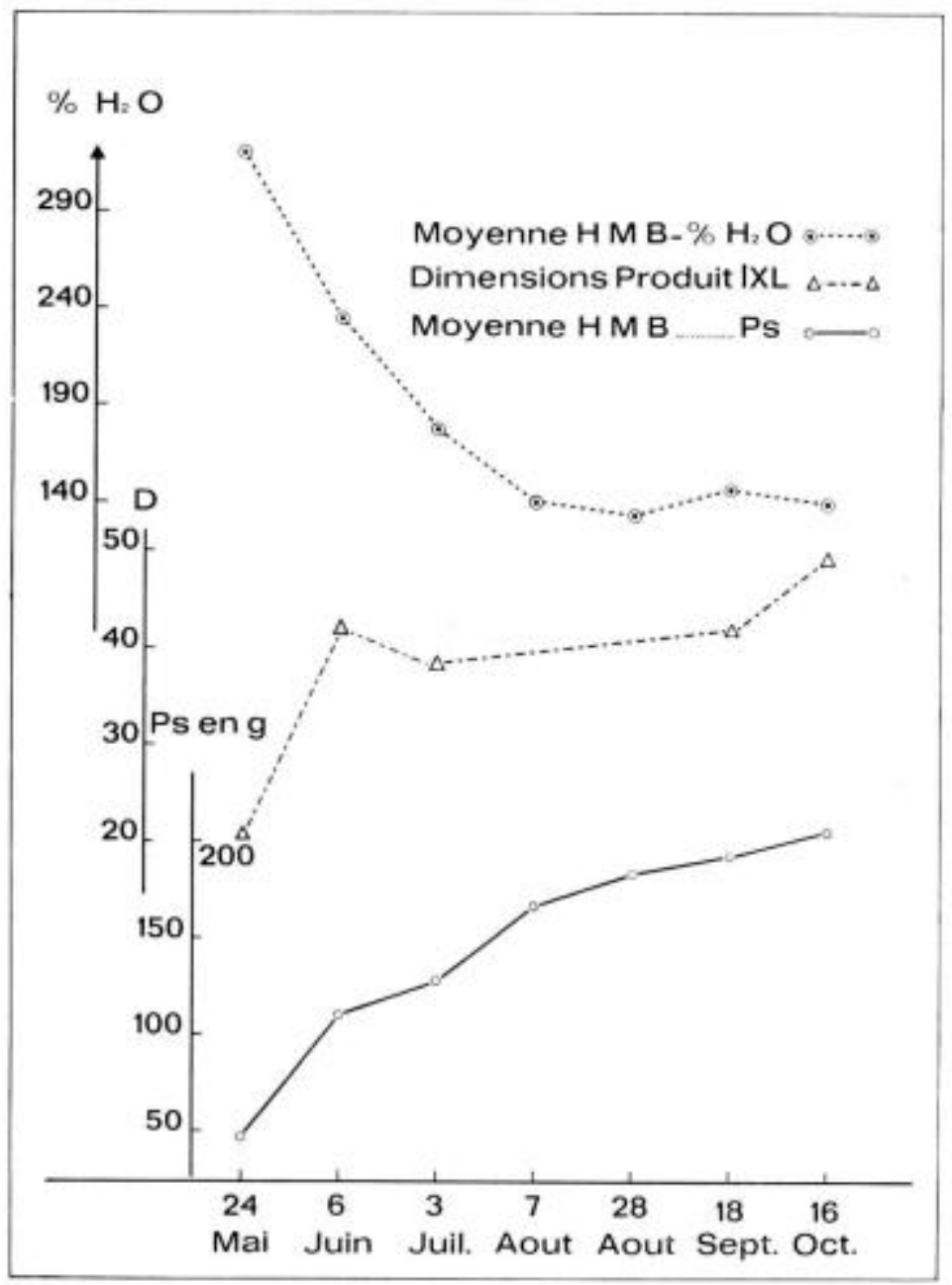

Fig. 2. - Synthèse des résultats 1967

Les différences entre positions d'échantillonnage conduisent d'autre part à fournir des critères de différenciation entre feuilles d'ombre et feuilles de lumière. Les observations sur les gourmands, en fournissant un étage supplémentaire de mesure, confirment assez bien ces critères. Ainsi les feuilles de lumière sont-elles plus petites, plus pauvres en eau et plus riches en matière sèche que les feuilles d'ombre, ce qui confirme dans le cas du chêne, les conclusions apportées pour d'autres essences. 
Il n'apparaît enfin aucune liaison significative entre les teneurs en eau individuelles des arbres, ou leur poids de 1000 feuilles sèches avec leur taille en diamètre ou hauteur. Cette absence de corrélation s'explique par l'influence d'un très grand nombre de facteurs sur la croissance d'un arbre. Cependant, si ces liaisons n'existent pas au niveau individuel sur une station, nous pensons pouvoir établir des corrélations poids sec ou teneur en eau avec la production globale en comparant diverses stations (étude en cours de rédaction).

\subsection{Problèmes d'échantillonnage}

II n'est pas sans intérêt de constater que les variations observées dans la croissance des feuilles peuvent interférer dans la pratique des échantillonnages foliaires en vue d'analyses de nutrition. Remarquons aussi que les mensurations d'ordre purement physique que nous avons définies pourront servir elles-mêmes d'indications dans des comparaisons de stations.

La difficulté majeure pour l'échantillonnage vient certainement des différences qui se manifestent d'une année à l'autre. Dans une étude de production, il serait ainsi prudent de réaliser tous les prélèvements sur une même année et si possible en fin août. Un échantillonnage sur position médiane, sur dix arbres pourrait convenir à la fois pour les teneurs en eau et pour les poids secs. Pour ces derniers, la meilleure estimation serait donnée par un prélèvement en fin de saison ; nous avons cependant vérifié statistiquement qu'il était possible d'opérer en août. Nous reprendrons la notion d'erreur d'échantillonnage à propos de nos études de stations.

\section{4. - ANALYSES CHIMIQUES}

Les cinq éléments que nous avons retenus ont été analysés pour 1966, à chaque époque, pour les trois positions d'échantillonnage et pour chacun des dix arbres. Pour 1967, les analyses n'ont porté que sur les quatre premières dates et ne fournissent pour l'instant que des possibilités de vérifications ( ${ }^{1}$ ).

Les résultats d'analyses sont exprimés à la fois en $\%$ du poids sec et en valeur absolue, c'est-à-dire en poids de l'élément pour 1000 feuilles. De cette façon, nous pourrons détecter au moins une partie des éventuels transferts des feuilles vers les organes de réserve (Qureshi et Srisvastava, 1966). A priori, sur le chêne, essence à feuillage annuel, on peut s'attendre à ce que ces phénomènes de transfert, ou translocation prennent des allures différentes de celles observées sur résineux.

Par analogie avec ce qui précède, nous commenterons d'abord successivement les divers résultats pour déboucher sur une conclusion globale, essentiellement consacrée aux problèmes d'échantillonnage.

(1) L.es analyses ont porté sur les feuilles entières, ce'est-â-díre limbes et pétioles. Sur chène pédonculé, le poids relatif du petiole est en effet très faible par rapport à celui du limbe (moins de I les différences analytiques entre limbes et pétioles scnt assez peu prononcées. 


\section{1. - Variations moyennes pour les dix arbres}

\subsection{Evolution saisonnière des teneurs en éléments minéraux des feuilles}

Pour suivre au mieux cette évolution, les résultats concernant les trois positions d'échantillonnage ont été regroupés. Dans un premier temps, seule la date d'échantillonnage interviendra donc comme facteur de variation. De cette façon, pour une date quelconque, nous disposons de trente échantillons par élément dosé sauf en octobre 1966 où les prélèvements n'ont été réalisés que sur cinq arbres, soit 15 échantillons par éléments. Pour chacun des éléments, analyse de variance et ajustement de courbe par régression polynomiale pondérée permettent de définir le sens des variations saisonnières. Les figures 3 à 7 avec leurs notes explicatives, résument parfaitement ces résultats. En outre, le tableau 5 regroupant les données partielles 1966 et 1967 concernant les poids de chacun des éléments pour 1000 feuilles met en relief les phénomènes de stockage et de transfert d'éléments au cours de la saison de végétation. Nous n'avons malheureusement pu obtenir un panorama complet de ces phénomènes sur une année entière, aussi avons-nous essayé de tirer des conclusions à partir des résultats de la deuxième moitié de la saison 1966 et de ceux de la première moitié 1967. Cette opération critiquable dans le sens où les deux années ne sont pas

\section{TABLEAU 5}

Poids d'éléments contenus dans 1000 feuilles exprimées en $g$ Variations en fonction de la date et de la position d'échantillonnage

(Les années 1966 et 1967 sont inversées dans le tableau de façon à recomposer imparfaitement une saison de végétation)

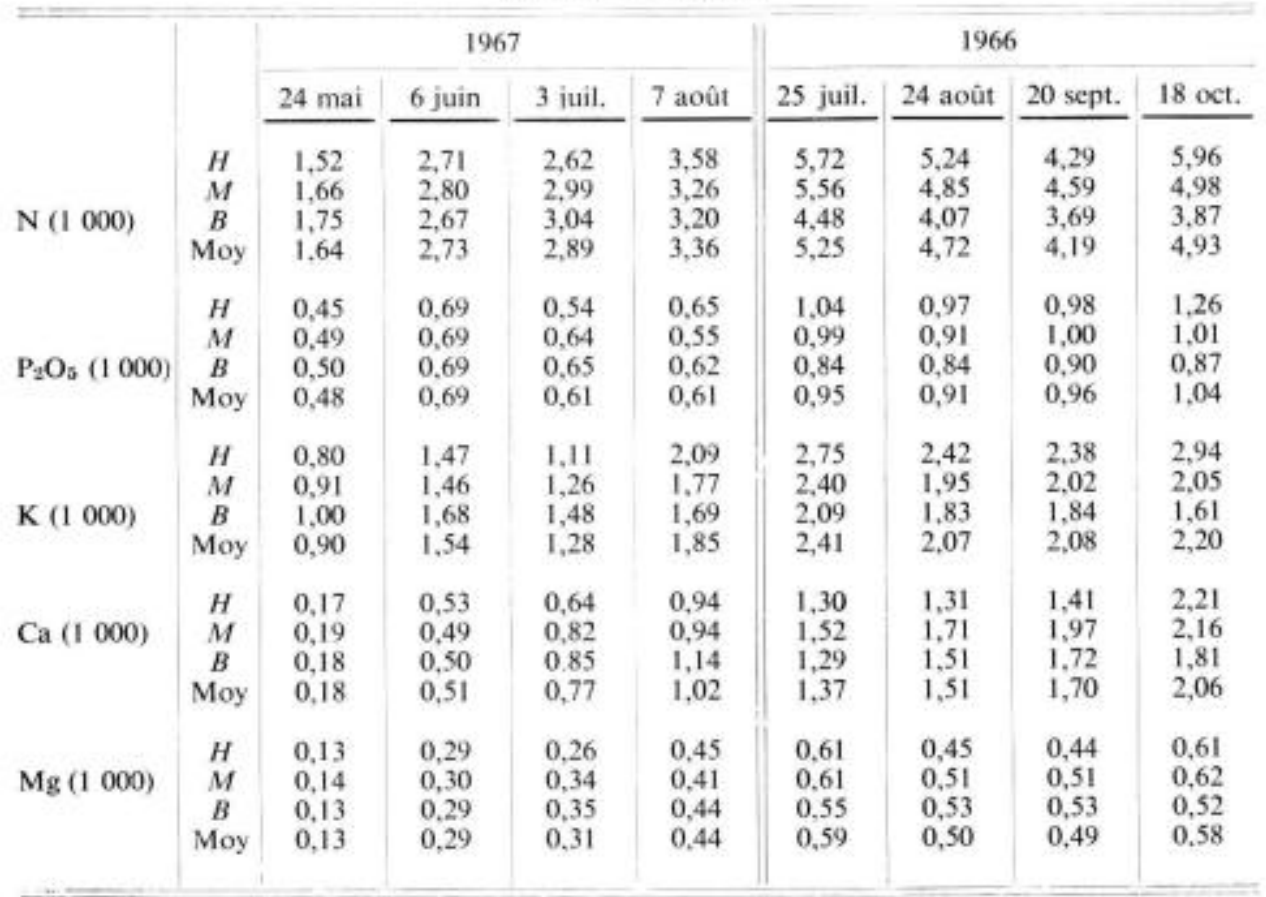


comparables entre elles, permet cependant quelques observations. Nous pouvons ainsi constater qu'à partir du mois d'août, la phase de stockage rapide des éléments dans la matière foliaire est pratiquement terminée sauf pour $\mathrm{Ca}$.

Nous avons résumé ci-dessous les observations que nous avons pu faire à propos de chaque élément. Remarquons pourtant dès maintenant que l'analyse de variance permet d'affirmer que la date intervient de facon significative sur les teneurs en éléments minéraux des feuilles de chêne, du moins les cinq éléments dosés N, P, K, $\mathrm{Ca}$ et dans une moindre mesure $\mathrm{Mg}$.

La concentration de lazote $(\% \mathrm{~N})$ (fig. 3) diminue de façon pratiquement continue de juin à octobre. Cette décroissance du $\% \mathrm{~N}$ en fonction de la date peut être représentée par une cubique qui laisse entrevoir pour 1966 une chute rapide du \% N

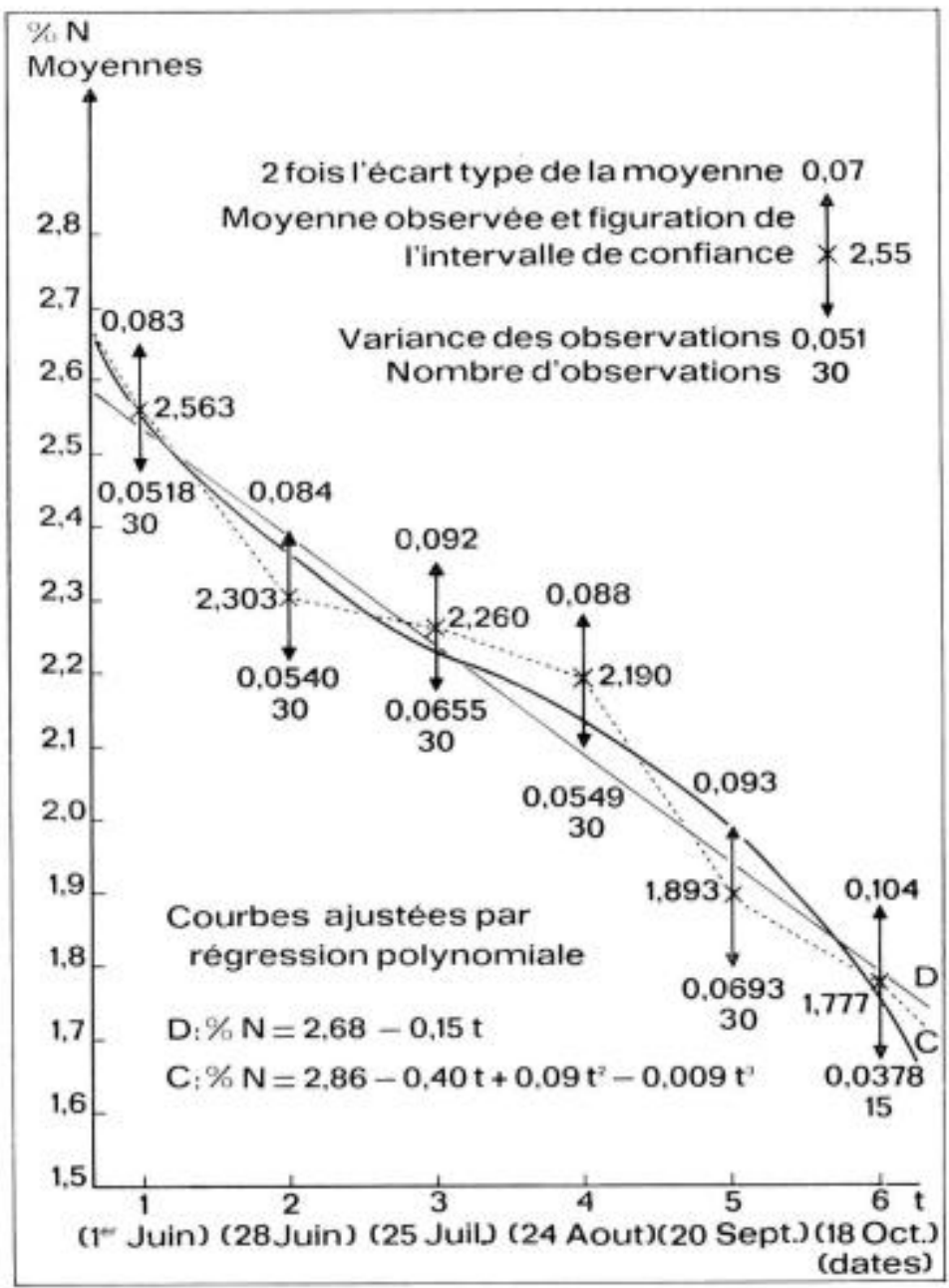

FIG, 3. - Variations saisonnières du \% $N$ dans les feuilles de chëne. Moyenne des trois positions d'échantillonnage 1966 
en tout début de saison. Les premiers résultats 1967 (tableau 7) confirment cette tendance, n'oublions pas en effet, qu'en 1967, les prélèvements ont démarré plus tôt après le débourrage qu'en 1966.

Il ne faut cependant pas perdre de vue que les courbures de la cubique sont peu prononcées ; en effet, une droite pourrait, en 1966, représenter la variation de \% $\mathrm{N}$ à partir du ler juin. II semble ainsi que le $\% \mathrm{~N}$ après une courte phase de décroissance brutale en début de saison diminue de façon presque constante de juin à octobre mis à part un pallier peu prononcé en juillet et août.

Les résultats partiels 1966 et 1967 concernant les poids d'azote pour 1000 feuilles, ou N (1000), permettent de penser qu'après une période d'accumulation, l'azote a tendance à migrer pour une partie, vraisemblablement vers les organes de

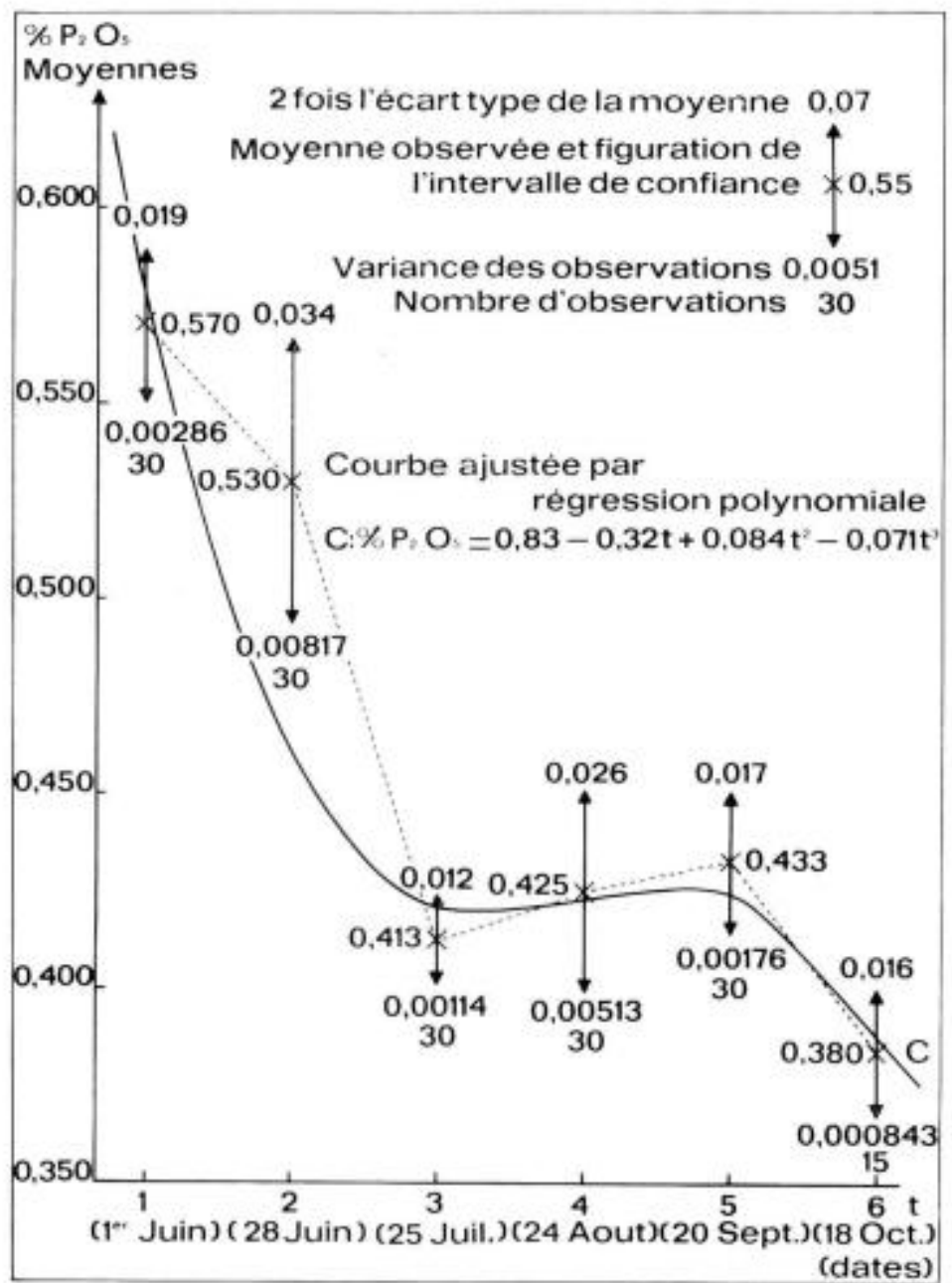

Fic. 4. - Variations saisonnières du \% $\mathrm{P}_{2} \mathrm{O}_{5}$ dans les feuilles de chêne. Moyenne des trois positions d'echantillonnage 1966 
réserves. Ainsi en 1966, du 25 juillet au 20 septembre, $20 \%$ de l'azote foliaire a pu disparaitre. En fin de saison, au contraire, on assiste à une nouvelle phase d'accumulation.

Les variations de concentration en phosphore et potassium $\left(\% \mathrm{P}_{2} \mathrm{O}_{5}, \% \mathrm{~K}\right)$ en fonction de la date sont également représentées par des cubiques (fig. 4 et 5 ). Les concentrations diminuent globalement avec l'âge des feuilles mais à la différence de l'azote, on peut observer pour ces deux éléments, la présence d'un pallier accentué au milieu de la saison de végétation. Il semble en outre qu'après la phase de décroissance brutale, les $\% \mathrm{P}_{2} \mathrm{O}_{5}$ et $\% \mathrm{~K}$ remontent légèrement ; cette tendance est nette pour $\mathrm{K}$ à la fois en 1966 et en 1967.

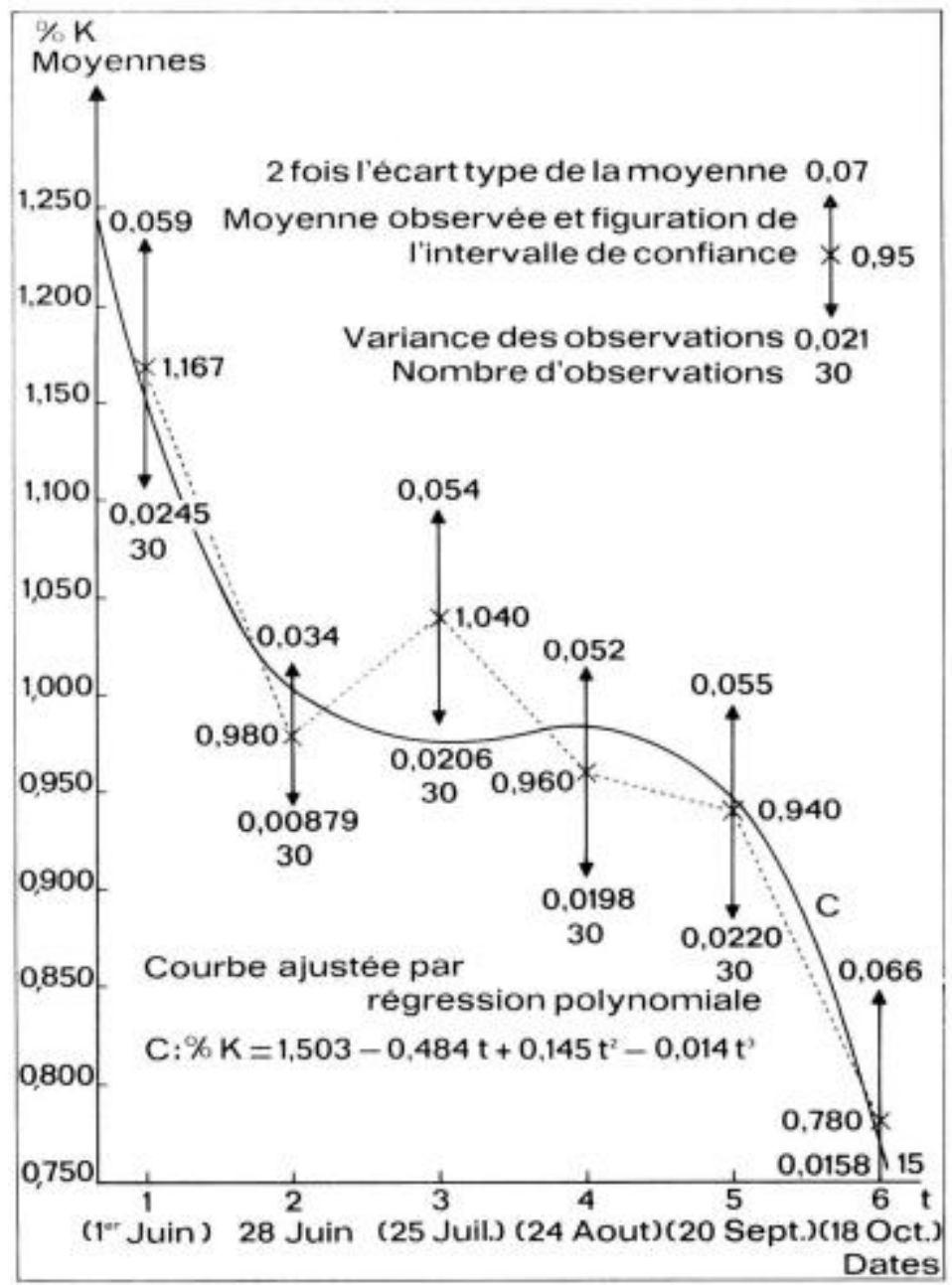

FıG. 5. - Variations saisonnières du $\% K$ dans les feuilles de chêne. Moyenne des trois positions d'échantillonnage 1966 
Les poids d'éléments pour 1000 feuilles, $\mathrm{P}_{2} \mathrm{O}_{5}\left(\begin{array}{l}1 \\ 1000)\end{array}\right)$ et $\mathrm{K}(1000)$ laissent apparaître de leur côté des tendances légèrement contradictoires. Tandis que $\mathrm{P}_{2} \mathrm{O}_{5}$ (1 000) augmente jusqu'en juillet pour rester relativement stable par la suite, on voit $\mathrm{K}$ (1 000) s'élever jusqu'au milieu de la saison de végétation, mais diminuer un peu en août et septembre. On peut ainsi déceler des transferts de potassium en milieu de saison, alors que le phosphore on ne peut rien affirmer.

Le magnésium suit une évolution particulière (fig. 6). De façon générale, on assiste à une décroissance faible et peu significative du \% Mg. Les courbures de la cubique représentant les variations du $\% \mathrm{Mg}$ sont par ailleurs inverses de celles observées pour $\mathrm{N}, \mathrm{P}$ et $\mathrm{K}$. Tout se passe comme si un pallier pour $\mathrm{Mg}$ voulait se dessiner en

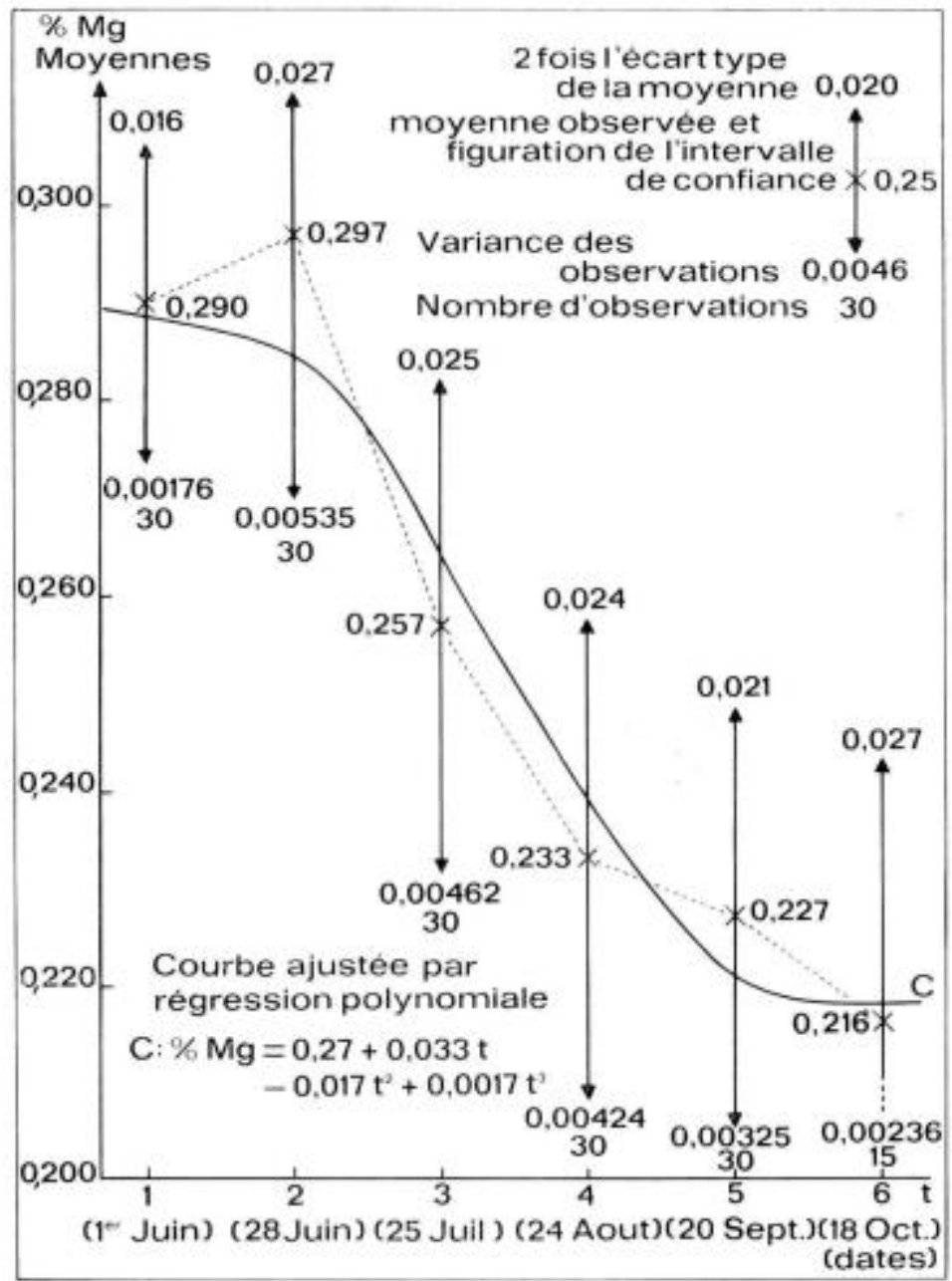

FIG. 6. - Variations saisonmières du \% Mg dans les feuilles de chêne. Moyenne des trois positions d'échantillonnage 1966 
début de saison. En fait, retenons une décroissance générale mais faible du $\% \mathrm{Mg}$ en fonction de la date.

L'indice $\mathrm{Mg}$ (1 000) quant à lui laisserait supposer de légers transferts de magnésium entre juillet et septembre.

Il reste le cas du calcium qui, contrairement aux autres éléments dosés, voit sa concentration augmenter du simple au double de juin à septembre-octobre (fig. 7). La parabole est la courbe la mieux ajustée au sens des variations.

L'accroissement continu de Ca ( 1000 ) illustre bien cette accumulation du calcium dans les feuilles.

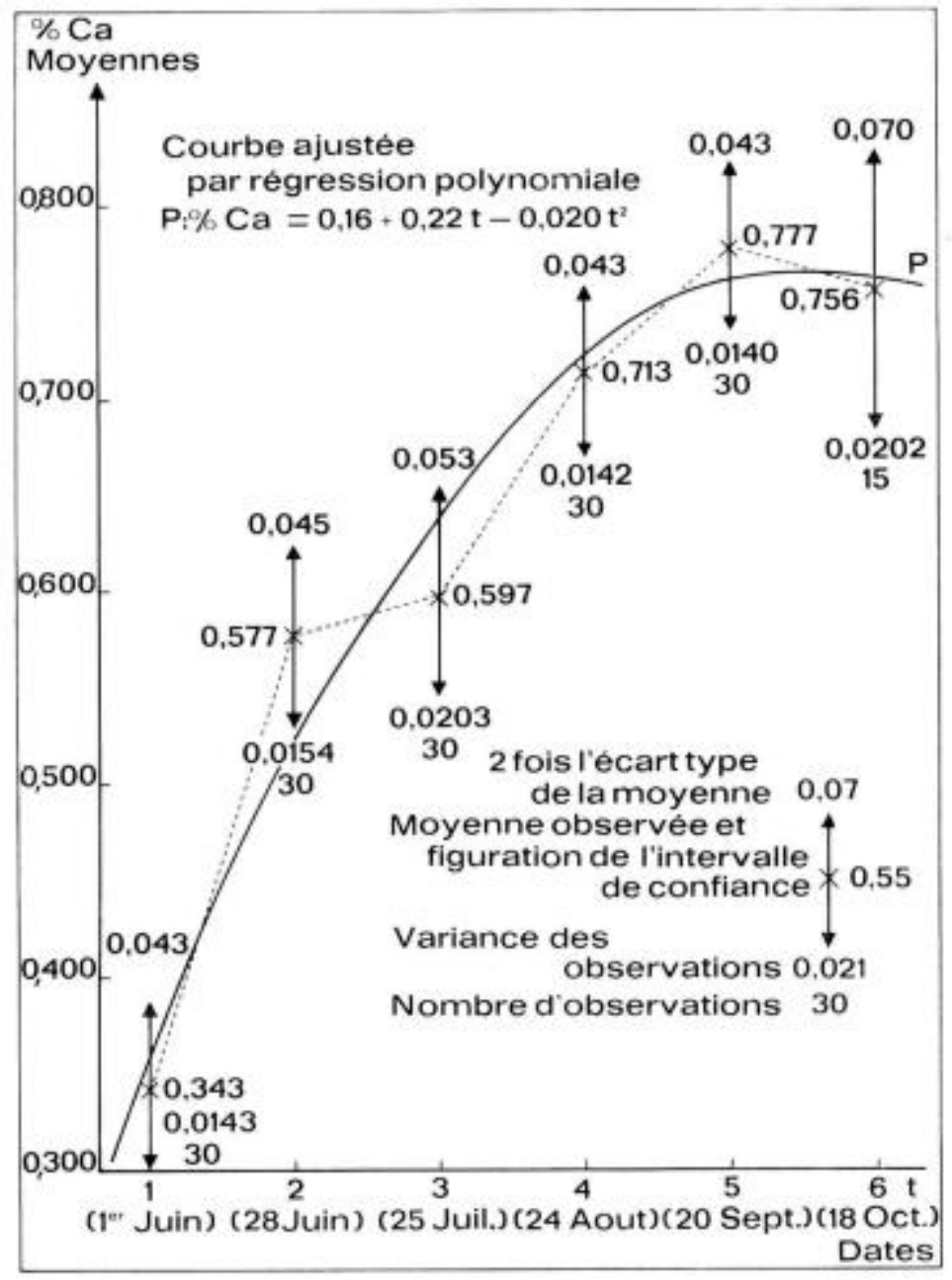

FIG. 7. - Variations saisonnières du \% Ca dans les feuilles de chêne. Moyenne des trois positions d'échantillonnage 1966 
Nos résultats relatifs aux variations de concentration en éléments concordent dans leur ensemble avec les quelques observations qu'ont pu faire divers auteurs (GUHA et Mitchell, 1966 ; FiedLer et HoHNE, 1965). Notre travail nous permet cependant de proposer des conclusions plus élaborées en ce qui concerne les cinq éléments que nous avons analysés.

Les $\% \mathrm{~N}, \mathrm{P}$ et $\mathrm{K}$ diminuent fortement du début à la fin de la saison de végétation : pour chacun de ces éléments, on observe une chute brutale de concentration au moment du développement actif des feuilles en début de saison. Cependant, la durée de ces phases de baisse rapide dépend de l'élément considéré :

- pour l'azote, elle est excessivement courte et se place sensiblement pendant la période de croissance rapide en dimensions des feuilles (fin mai, début juin).

- pour le potassium, elle couvre le mois de juin et correspond à la période de décroissance rapide des $\% \mathrm{H}_{2} \mathrm{O}$ (tableau 1, 1966). Nous verrons plus loin que les corrélations $\% \mathrm{H}_{2} \mathrm{O}-\% \mathrm{~K}$ confirment cette constatation.

- pour le phosphore, elle s'étale jusqu'en fin juillet, début août,

Par ailleurs, pour $\mathrm{N}$ et $\mathrm{K}$, les translocations que nous avons pu isoler s'effectueraient au cours de la saison de végétation et non pas au moment de la sénescence comme le signalaient GuHA et Mrtchel. pour K.

Enfin, le \% Ca ainsi que Ca $(1000)$ augmentent de façon continue de mai à septembre-octobre. Pour le magnésium, nous avons pu relever au contraire une relative stabilité de concentration, la décroissance du \% $\mathrm{Mg}$ est en effet assez faible entre mai et octobre ; celà laisserait supposer que cet élément suivrait grossièrement les fluctuations du poids de matière sèche.

Dans un autre domaine, les comparaisons entre les années 1966 et 1967 présentent quelque intérêt (tableaux 6 et 7). Le caractère partiel des résultats 1967 ne permet pas de juger des différences de concentration entre les deux années, sauf peut-être pour l'azote dont le taux juillet-août semble sensiblement plus bas en 1967 qu'en 1966. Par contre, les gros écarts observés entre les " poids secs de 1000 feuilles " des deux années conduisent logiquement à des " poids d'éléments pour 1000 feuilles » assez différents. Il suffit pour s'en persuader de comparer ces indices pour les 25 juillet 1966 et le 7 août 1967 (tableau 5).

\subsection{Influence de la position d'échantillonnage sur les concentrations}

Les tableaux 5 et 6 regroupent pour chaque date les données 1966 relatives aux trois positions d'échantillonnage. Remarquons au départ que pour chaque position, les variations de concentration en fonction de l'époque reproduisent assez fidèlement les variations moyennes décrites ci-dessus.

Une analyse de variance globale, réalisée pour l'ensemble des dates, permet d'affirmer que la position intervient de façon significative sur les concentrations des cinq éléments dosés. Cependant I'analyse de variance, effectuée date par date nuance un peu cette affirmation : les différences significatives n'apparaissent pas pour toutes les dates : les écarts dus à la position sont parfois faibles pour une date donnée. 
La concentration en azote est ainsi globalement significativement plus élevée dans le bas que dans le haut de la cime, mais le seul écart significatif par date, apparaît le 28 juin. Notons quand même que pour toutes les dates, les écarts B-H sont positifs. Au tableau 5, pour 1966 les N (1 000) en fonction de la position montrent la tendance inverse, ce qui s'explique naturellement par nos remarques précédentes au sujet des variations du Ps en fonction de la position dans la cime.

\section{TABLEAU 6}

1966 - Concentrations en éléments minéraux en fonction de la date et de la position d'échantillonnage

Concentrations en $\%$ du poids sec

$H=$ Tiers supérieur de la couronne

$M=$ Tiers médian de la couronne

$B=$ Tiers inférieur de la couronne

Moy $=$ Moyenne des trois positions $H, M, B$.

\begin{tabular}{|c|c|c|c|c|c|c|c|}
\hline Elément & Position & $1^{e r}$ juin & 28 juin & 25 juil. & 24 août & 20 sept. & 18 oct. \\
\hline$\% \mathrm{~N}$ & $\begin{array}{c}H \\
M \\
B \\
\text { Moy }\end{array}$ & $\begin{array}{l}2,563 \\
2,553 \\
2,575 \\
2,563\end{array}$ & $\begin{array}{l}2,149^{*} \\
2,384 \\
2,381 \\
2,303\end{array}$ & $\begin{array}{l}2,166 \\
2,304 \\
2,310 \\
2,260\end{array}$ & $\begin{array}{l}2,152 \\
2,213 \\
2,205 \\
2,190\end{array}$ & $\begin{array}{l}1,801 \\
1,966 \\
1,911 \\
1,893\end{array}$ & $\begin{array}{l}1,640 \\
1,844 \\
1,848 \\
1,777\end{array}$ \\
\hline$\% \mathrm{P}_{2} \mathrm{O}_{5}$ & $\begin{array}{c}H \\
M \\
B \\
\text { Moy }\end{array}$ & $\begin{array}{l}0,566 \\
0,564 \\
0,585 \\
0,570\end{array}$ & $\begin{array}{l}0,528 \\
0,535 \\
0,521 \\
0,530\end{array}$ & $\begin{array}{l}0,392^{\circ} \\
0,412 \\
0,436 \\
0,413\end{array}$ & $\begin{array}{l}0,398^{*} \\
0,413 \\
0,465 \\
0,425\end{array}$ & $\begin{array}{l}0,412^{*} \\
0,428 \\
0,464 \\
0,433\end{array}$ & $\begin{array}{l}0,348^{*} \\
0,376 \\
0,414 \\
0,380\end{array}$ \\
\hline$\% \mathrm{~K}$ & $\begin{array}{c}H \\
M \\
B \\
\text { Moy }\end{array}$ & $\begin{array}{l}1,113 \\
1,126 \\
1,255 \\
1,167\end{array}$ & $\begin{array}{l}0,981 \\
0,942 \\
1,018 \\
0,980\end{array}$ & $\begin{array}{l}1,040 \\
0,996 \\
1,077 \\
1,040\end{array}$ & $\begin{array}{l}0,996 \\
0,891 \\
0,994 \\
0,960\end{array}$ & $\begin{array}{l}1,002 \\
0,866 \\
0,953 \\
0,940\end{array}$ & $\begin{array}{l}0,810 \\
0,758 \\
0,770 \\
0,780\end{array}$ \\
\hline$\% \mathrm{Ca}$ & $\begin{array}{c}H \\
M \\
B \\
\text { Moy }\end{array}$ & $\begin{array}{l}0,335 \\
0,349 \\
0,340 \\
0,343\end{array}$ & $\begin{array}{l}0,454^{*} \\
0,576 \\
0,701 \\
0,577\end{array}$ & $\begin{array}{l}0,491^{*} \\
0,632 \\
0,665 \\
0,597\end{array}$ & $\begin{array}{l}0.538^{*} \\
0,782 \\
0,818 \\
0,713\end{array}$ & $\begin{array}{l}0,594^{*} \\
0,845 \\
0,889 \\
0,777\end{array}$ & $\begin{array}{l}0,608^{*} \\
0,800 \\
0,862 \\
0,756\end{array}$ \\
\hline$\% \mathrm{Mg}$ & $\begin{array}{c}H \\
M \\
B \\
\text { Moy }\end{array}$ & $\begin{array}{l}0,291 \\
0,304 \\
0,281 \\
0,290\end{array}$ & $\begin{array}{l}0,274 \\
0,294 \\
0,333 \\
0,297\end{array}$ & $\begin{array}{l}0,230 \\
0,253 \\
0,286 \\
0,257\end{array}$ & $\begin{array}{l}0,187^{*} \\
0,232 \\
0,287 \\
0,233\end{array}$ & $\begin{array}{l}0,184^{*} \\
0,217 \\
0,276 \\
0,227\end{array}$ & $\begin{array}{l}0,168^{*} \\
0,230 \\
0,250 \\
0,216\end{array}$ \\
\hline
\end{tabular}

L'astérisque $\left({ }^{*}\right)$ situé dans l'angle supérieur droit d'une case indique qu'il y a différence significative entre $H$ et $B$.

La concentration en phosphore est également plus élevée dans le bas que dans le haut du houppier. Les écarts significatifs par date sont cependant plus fréquents que pour l'azote, puisqu'ils apparaissent régulièrement à partir de fin juillet. Les $\mathrm{P}_{2} \mathrm{O}_{5}(1000)$ en fin de saison n'en restent pas moins plus élevés dans le haut que dans le bas. Le potassium de son côté, semble suivre une voie originale. En effet, globalement la position d'échantillonnage intervient significativement sur le $\% \mathrm{~K}$, tandis que l'analyse de variance par date ne révèle aucune différence significative. Il semble néanmoins possible d'observer une inversion au cours de la saison de végétation : le $\% \mathrm{~K}$ est 
d'abord plus fort en bas qu'en haut pour tendre ensuite à devenir plus faible, quand la corrélation $\% \mathrm{H}_{2} \mathrm{O}-\% \mathrm{~K}$ n'est plus significative. Le tableau 7 relatif aux résultats 1967 confirmerait cette hypothèse.

\section{TABLEAU 7}

1967 - Concentrations en éléments minéraux en fonction de la date et de la position d'échantillonnage

Concentrations en $\%$ du poids sec

$H=$ Tiers supérieur de la couronne

$M=$ Tiers médian de la couronne

$B=$ Tiers inférieur de la couronne

Moy $=$ Moyenne des trois positions $H, M, B$.

\begin{tabular}{|c|c|c|c|c|c|}
\hline Elément & Position & 24 mai & 6 juin & 3 juil. & 7 août \\
\hline$\% \mathrm{~N}$ & $\begin{array}{c}H \\
M \\
B \\
\text { Moy }\end{array}$ & $\begin{array}{l}3,566 \\
3,439 \\
3,566 \\
3,523\end{array}$ & $\begin{array}{l}2,420 \\
2,522 \\
2,431 \\
2,457\end{array}$ & $\begin{array}{l}2,243 \\
2,303 \\
2,282 \\
2,276\end{array}$ & $\begin{array}{l}1,936 \\
2,013 \\
2,121 \\
2,023\end{array}$ \\
\hline$\% \mathrm{P}_{2} \mathrm{O}_{5}$ & $\begin{array}{c}H \\
M \\
B \\
\text { Moy }\end{array}$ & $\begin{array}{l}1,051 \\
1.020 \\
1.024 \\
1.031\end{array}$ & $\begin{array}{l}0,597 \\
0,624 \\
0,631 \\
0,617\end{array}$ & $\begin{array}{l}0,459 \\
0,490 \\
0,492 \\
0,480\end{array}$ & $\begin{array}{l}0,352 \\
0,341 \\
0,413 \\
0,368\end{array}$ \\
\hline$\% \mathrm{~K}$ & $\begin{array}{c}H \\
M \\
B \\
\text { Moy }\end{array}$ & $\begin{array}{l}1,890 \\
1,894 \\
2,048 \\
1,944\end{array}$ & $\begin{array}{l}1,311 \\
1,315 \\
1,530 \\
1,385\end{array}$ & $\begin{array}{l}0,951 \\
0,973 \\
1,113 \\
1,012\end{array}$ & $\begin{array}{l}1,134 \\
1,092 \\
1,121 \\
1,115\end{array}$ \\
\hline$\% \mathrm{Ca}$ & $\begin{array}{c}H \\
M \\
B \\
\text { Moy }\end{array}$ & $\begin{array}{l}0,395 \\
0,393 \\
0,378 \\
0,388\end{array}$ & $\begin{array}{l}0,472 \\
0,443 \\
0,451 \\
0,455\end{array}$ & $\begin{array}{l}0,550 \\
0,633 \\
0,638 \\
0,607\end{array}$ & $\begin{array}{l}0,508 \\
0,581 \\
0,757 \\
0,615\end{array}$ \\
\hline$\% \mathrm{Mg}$ & $\begin{array}{c}H \\
M \\
B \\
\text { Moy }\end{array}$ & $\begin{array}{l}0,297 \\
0,295 \\
0,276 \\
0,289\end{array}$ & $\begin{array}{l}0,261 \\
0,266 \\
0,264 \\
0,264\end{array}$ & $\begin{array}{l}0,224 \\
0,259 \\
0,263 \\
0,248\end{array}$ & $\begin{array}{l}0,246 \\
0,251 \\
0,294 \\
0,264\end{array}$ \\
\hline
\end{tabular}

C'est pour le calcium que les écarts entre haut et bas du houppier sont les plus grands. A partir de juin, pour chaque date, les différences sont fortement significatives. En moyenne, les feuilles du bas contiennent 30 à $35 \%$ de Ca de plus que celles du haut.

Le magnésium, enfin, voit également sa concentration plus élevée dans les feuilles du bas que dans celles du haut, cette tendance étant très marquée dès la fin août. Ainsi, en fait, si le \% Mg varie peu avec la date, la distribution du magnésium dans la cime n'est néanmoins pas homogène.

Nous pouvons retenir que globalement la position d'échantillonnage intervient sur les concentrations en $\mathrm{N}, \mathrm{P}, \mathrm{K}$ et $\mathrm{Mg}$ en complétant cette conclusion des remarques suivantes :

- en début de saison de végétation, aucune différence significative n'apparait entre positions, 
- Les concentrations en N,P, Ca et Mgsont plus fortes dans les feuilles d'ombre que dans les feuilles de lumière. Les quelques vérifications que nous avons pu faire sur gourmands confirment d'ailleurs ce résultat. Les écarts moyens absolus, à partir de juillet entre haut et bas du houppier pour $\mathrm{N}, \mathrm{P}_{2} \mathrm{O}_{5}, \mathrm{Ca}$ et $\mathrm{Mg}$ sont respectivement de l'ordre de $0,17 \%, 0,06 \%, 0,25 \%$ et $0,1 \%$ soit des écarts relatifs de $8,5 \%, 14 \%$, $35 \%$ et $40 \%$.

- La position d'échantillonnage n'induit pour une date quelconque aucune différence significative dans les concentrations en potassium. II semble cependant que les mécanismes d'alimentation potassique des feuilles d'ombre et de lumière dépendent étroitement de la date. L'extrême mobilité du potassium dans les tissus végétaux se trouve ainsi une fois de plus confirmée, d'autant plus confirmée que d'éventuels transferts de $\mathrm{K}$ pourraient se réaliser en fin de saison des feuilles du bas vers celles du haut (tableau 5).

- Les poids d'éléments contenus dans 1000 feuilles dépendent à la fois des concentrations et des poids secs de 1000 feuilles ou Ps. Rappelons que les feuilles du haut ont un Ps supérieur à celles du bas. Ainsi, en fin de saison, 1000 feuilles de lumière contiennent davantage de N, P et K que 1000 feuilles d'ombre. Pour Ca et $\mathrm{Mg}$, on observe le contraire et ceci s'explique par le fait que les différences de concentration en $\mathrm{Ca}$ et $\mathrm{Mg}$ entre bas et haut du houppier sont telles qu'elles annulent les écarts observés sur les Ps.

On pourrait tenter dexpliquer les différences de concentration entre haut et bas de la cime par le seul fait des effers de dilution. Cependant, les seules observations à propos du potassium suffisent à démontrer l'influence de phénomènes physiologiques sur la distribution des éléments minéraux dans le feuillage ; on voit, en effet, le \% K évoluer de façon assez indépendante des Ps. En outre, les écarts relatifs "bas-sommet $»$ des autres éléments dosés sont loin d'être voisins ce qui serait le cas si un simple effet de dilution intervenait. En définitive, il semble évident que les différences de concentrations entre feuilles d'ombre et feuilles de lumière sont liées à la fois à des mécanismes physiologiques et aux effets de dilution dans la matière sèche.

On peut, pour affiner cette observation, comparer les écarts relatifs entre $\mathrm{H}$ et B pour chaque élément, aux 25 ou $30 \%$ qui représentent l'écart relatif existant entre les Ps, H et B de fin de saison. A partir de ces comparaisons, il semble possible de penser qu'il y a alimentation préférentielle des feuilles de lumière par rapport aux feuilles d'ombre en $\mathrm{N}, \mathrm{P}$ et de toute évidence en $\mathrm{K}$. Pour Ca et $\mathrm{Mg}$, la tendance serait inverse.

\subsection{Conchusion partielle}

L'étude des variations saisonnières moyennes des teneurs en éléments minéraux des feuilles nous a conduit à émettre quelques observations à propos des mécanismes de distribution des éléments dans le feuillage. D'une façon générale, il apparait que pour $\mathrm{N}, \mathrm{P}, \mathrm{K}$ et $\mathrm{Mg}$. l'accumulation dans la matière foliaire est plus rapide en début qu'en fin de saison. Cependant, le stockage en ces éléments reste plus actif en fin de saison dans les feuilles du haut que dans celles du bas ; cette remarque 
confirme le fait que les feuilles du haut, maintiennent une activité physiologique plus longtemps que celles du bas. Pour le calcium, on assiste à une accumulation progressive et presque constante dans l'ensemble de la masse foliaire.

Il est difficile de tirer un parti valable de ces observations sans faire une étude complexe portant sur le flux d'éléments minéraux au travers des divers organes végétatifs pendant la saison de végétation ; les feuilles ne présentent en effet qu'un aspect très localisé de la nutrition minérale. Nous avons néanmoins mis en évidence les variations de concentrations folaires en $\mathrm{N}, P, K, \mathrm{Ca}$ et $\mathrm{Mg}$, en fonction à la fois du temps et de la hauteur dans la cime.

Ces variations ne sont pas uniquement provoquées par des effets de dilution ; l'intervention de processus physiologiques apparait nettement pour les cinq éléments dosés. En outre, si les phénomènes de transfert, ou translocation, sont difficilement révélés par notre méthode de travail, nous pouvons pourtant affirmer qu'ils interviennent beaucoup plus tôt dans le cours de la saison de végétation que ne le pensaient divers auteurs.

\section{2. - Informations complémentaires apportées par les analyses chimiques}

En tenant compte maintenant des teneurs individuelles en éléments minéraux de chacun de nos dix arbres, nous allons pouvoir dégager des informations supplémentaires.

Il faut déjà noter que les variations individuelles de concentration en fonction de l'époque d'échantillonnage sont assez disparates. Les courbes de variations individuelles sont du même type que les courbes de variations moyennes décrites précédemment, mais présentent entre elles des décalages relativement importants tant dans le sens des abcisses que dans celui des ordonnées. Nous avons d'ailleurs vérifié que ces décalages n'étaient pas le fait du hasard : les corrélations entre les teneurs 1966 et 1967 pour chacun des éléments dosés sont fortement significatives. Ainsi par exemple, les coefficients de corrélations entre concentrations pour septembre 1966 et août 1967 sont respectivement de $0,50,0,43,0,56,0,67,0,40$ pour $\mathrm{N}, \mathrm{P}, \mathrm{K}$, Ca et $\mathrm{Mg}$ (28 d.d.1.). Cette hétérogénéité dans le comportement individuel laisse penser que l'influence des caractères génétiques sur la nutrition minérale est loin d'être négligeable.

Sur un tout autre plan, nous avons cherché à établir les diverses liaisons qui pourraient exister au niveau individuel, d'une part entre les éléments pris deux à deux, d'autre part entre les éléments et les diverses caractéristiques physiques que nous avons définies. C'est l'ensemble de ces résultats que nous présentons ci-après.

\subsection{Corrélations teneurs en éléments minéraux et teneur en eau}

Ces corrélations sont représentées sur la figure 8 par des diagrammes schématiques. Les liaisons les plus denses se manifestant en début et en fin de saison, on peut supposer que les processus physiologiques jouant sur l'eau et les éléments 
minéraux, interviennent le plus efficacement pendant ces périodes $\left({ }^{1}\right)$. En milieu de saison, les facteurs de la nutrition seraient alors relativement passifs ; un réajustement physiologique se réaliserait seulement au moment de la sénescence. Ce cycle coïnciderait d'ailleurs avec les fluctuations de $\% \mathrm{H}_{2} \mathrm{O}$ : remarquons, en effet, que le minimum d'intensité de liaison, et notamment les liaisons avec le $\% \mathrm{H}_{2} \mathrm{O}$, se manifeste au moment même où les déficits de saturation en eau sont les plus forts.

\section{TABLEAU 8}

1966 - Rapports des éléments dosés pris deux à deux Variations en fonction de l'époque et de la position d'échantillonnage

Les éléments sont exprimés en $\%$ du poids sec :

$H=$ Tiers supérieur

$B=$ Tiers inférieur

Moy $=$ Rapport moyen $H, M, B$.

\begin{tabular}{|c|c|c|c|c|c|c|c|}
\hline Rapport & Position & $1^{e r}$ juin & 28 juin & 25 juil. & 24 août & 20 sept. & 18 oct. \\
\hline $\mathrm{N} / \mathrm{P}_{2} \mathrm{O}_{5}$ & $\begin{array}{c}H \\
B \\
\text { Moy }\end{array}$ & $\begin{array}{l}4,52 \\
4,40 \\
4,48\end{array}$ & $\begin{array}{l}4,07 \\
4,57 \\
4,36\end{array}$ & $\begin{array}{l}5,52 \\
5,30 \\
5,47\end{array}$ & $\begin{array}{l}5,40 \\
4,74 \\
5,16\end{array}$ & $\begin{array}{l}4,37 \\
4,12 \\
4,36\end{array}$ & $\begin{array}{l}4,71 \\
4,46 \\
4,69\end{array}$ \\
\hline $\mathrm{N} / \mathrm{K}$ & $\begin{array}{c}H \\
B \\
\text { Moy }\end{array}$ & $\begin{array}{l}2,30 \\
2,05 \\
2,20\end{array}$ & $\begin{array}{l}2,19 \\
2,33 \\
2,35\end{array}$ & $\begin{array}{l}2,08 \\
2,14 \\
2,17\end{array}$ & $\begin{array}{l}2,16 \\
2,21 \\
2,28\end{array}$ & $\begin{array}{l}1,80 \\
2,00 \\
2,02\end{array}$ & $\begin{array}{l}2,02 \\
2,40 \\
2,28\end{array}$ \\
\hline $\mathrm{N} / \mathrm{Ca}$ & $\begin{array}{c}H \\
B \\
\text { Moy }\end{array}$ & $\begin{array}{l}7,65 \\
7,57 \\
7,51\end{array}$ & $\begin{array}{l}4,73 \\
3,39 \\
4,08\end{array}$ & $\begin{array}{l}4,41 \\
3,47 \\
3,84\end{array}$ & $\begin{array}{l}4,00 \\
2,68 \\
3,17\end{array}$ & $\begin{array}{l}3,30 \\
2,15 \\
2,50\end{array}$ & $\begin{array}{l}2,69 \\
2,14 \\
2,37\end{array}$ \\
\hline $\mathrm{N} / \mathrm{Mg}$ & $\begin{array}{c}H \\
B \\
\text { Moy }\end{array}$ & $\begin{array}{l}8,80 \\
9,16 \\
8,78\end{array}$ & $\begin{array}{l}7,84 \\
7,15 \\
7,69\end{array}$ & $\begin{array}{l}9,42 \\
8,08 \\
8,87\end{array}$ & $\begin{array}{r}11,50 \\
7,68 \\
9,57\end{array}$ & $\begin{array}{l}9,80 \\
6,92 \\
8,59\end{array}$ & $\begin{array}{l}9,76 \\
7,39 \\
8,38\end{array}$ \\
\hline $\mathrm{K} / \mathrm{P}_{2} \mathrm{O}_{5}$ & $\begin{array}{c}H \\
B \\
\text { Moy }\end{array}$ & $\begin{array}{l}1,96 \\
2,14 \\
2,03\end{array}$ & $\begin{array}{l}1,85 \\
1,95 \\
1,85\end{array}$ & $\begin{array}{l}2,65 \\
2,47 \\
2,51\end{array}$ & $\begin{array}{l}2,50 \\
2,13 \\
2,26\end{array}$ & $\begin{array}{l}2,43 \\
2,05 \\
2,16\end{array}$ & $\begin{array}{l}2,32 \\
1,85 \\
2,06\end{array}$ \\
\hline $\mathrm{K} / \mathrm{Mg}$ & $\begin{array}{c}H \\
B \\
\text { Moy }\end{array}$ & $\begin{array}{l}3,82 \\
4,46 \\
3,99\end{array}$ & $\begin{array}{l}3,58 \\
3,05 \\
3,27\end{array}$ & $\begin{array}{l}4,52 \\
3,76 \\
4,07\end{array}$ & $\begin{array}{l}5,32 \\
3,46 \\
4,20\end{array}$ & $\begin{array}{l}5,44 \\
3,45 \\
4,29\end{array}$ & $\begin{array}{l}4,82 \\
3,08 \\
3,73\end{array}$ \\
\hline $\mathrm{K} / \mathrm{Ca}$ & $\begin{array}{c}H \\
B \\
\text { Moy }\end{array}$ & $\begin{array}{l}3,32 \\
3,69 \\
3,41\end{array}$ & $\begin{array}{l}2,16 \\
1,45 \\
1,74\end{array}$ & $\begin{array}{l}2,12 \\
1,62 \\
1,77\end{array}$ & $\begin{array}{l}1,85 \\
1,21 \\
1,40\end{array}$ & $\begin{array}{l}1,69 \\
1,07 \\
1,26\end{array}$ & $\begin{array}{l}1,33 \\
0,89 \\
1,05\end{array}$ \\
\hline $\mathrm{P}_{2} \mathrm{O}_{5} / \mathrm{Ca}$ & $\begin{array}{c}H \\
B \\
\text { Moy }\end{array}$ & $\begin{array}{l}1,68 \\
1,72 \\
1,67\end{array}$ & $\begin{array}{l}1,16 \\
0,74 \\
0,94\end{array}$ & $\begin{array}{l}0,80 \\
0,65 \\
0,70\end{array}$ & $\begin{array}{l}0,73 \\
0,56 \\
0,60\end{array}$ & $\begin{array}{l}0,69 \\
0,50 \\
0,57\end{array}$ & $\begin{array}{l}0,57 \\
0,48 \\
0,51\end{array}$ \\
\hline $\mathrm{P}_{2} \mathrm{O}_{5} / \mathrm{Mg}$ & $\begin{array}{c}H \\
B \\
\text { Moy }\end{array}$ & $\begin{array}{l}1,94 \\
2,08 \\
1,95\end{array}$ & $\begin{array}{l}1,92 \\
1,56 \\
1,76\end{array}$ & $\begin{array}{l}1,70 \\
1,52 \\
1,61\end{array}$ & $\begin{array}{l}1,56 \\
1,62 \\
1,65\end{array}$ & $\begin{array}{l}2,24 \\
1,68 \\
1,96\end{array}$ & $\begin{array}{l}2,07 \\
1,65 \\
1,78\end{array}$ \\
\hline $\mathrm{Ca} / \mathrm{Mg}$ & $\begin{array}{c}H \\
B \\
\text { Moy }\end{array}$ & $\begin{array}{l}1,15 \\
1,20 \\
1,16\end{array}$ & $\begin{array}{l}1,64 \\
2,10 \\
1,90\end{array}$ & $\begin{array}{l}2,13 \\
2,32 \\
2,31\end{array}$ & $\begin{array}{l}2,87 \\
2,85 \\
3,03\end{array}$ & $\begin{array}{l}3,23 \\
3,22 \\
3,45\end{array}$ & $\begin{array}{l}3,61 \\
3,44 \\
3,50\end{array}$ \\
\hline
\end{tabular}

(1) Le diagramme de corrélation đu 24 mai 1967 est exactement đu même type que celui du 1er juin 1966. 
Les corrélations positives qui apparaissent entre phosphore et azote en juin, juillet et octobre méritent d'être remarquées, de même que les corrélations négatives de juillet et août, entre $\mathrm{N}$ et $\mathrm{Ca}$.

Il reste en fait difficile d'interpréter pour l'instant le sens des diverses corrélations constatées. On peut quand même retenir qu'en début de saison, les rapports des éléments entre eux représentent certainement un équilibre physiologique. Cet équilibre serait ensuite partiellement rompu pour se reconstituer en partie au moment de la sénescence. La rupture n'est que partielle : nous avons établi, en effet, que la dilution, facteur passif, $n$ "intervenait jamais seule. La lecture du tableau 8 concernant l'évolution des rapports des éléments entre eux confirme cette hypothèse : tous les rapports où n'intervient pas $1 \mathrm{e} \%$ de Ca tendent à être très voisins, en juin et en octobre ; leurs variations entre ces dates sont d'ailleurs de faible importance. L'étude de ces rapports permet, en outre, d'introduire une remarque confirmant certains résultats précédents : les feuilles du haut n'ont pas tout à fait le même comportement vis-à-vis des éléments minéraux que celles du bas. II suffit, pour s'en persuader, de comparer entre eux les divers rapports pour feuilles du haut et feuilles du bas.

L'étude des rapports des éléments entre eux nous ouvre enfin une perspective intéressante. Les variations saisonnières des rapports où n'intervient pas le pourcentage de $\mathrm{Ca}$ sont en effet de faible amplitude ; d'autre part, les différences entre rapports du haut et du bas sont relativement petites. Ainsi, dans la mesure oú certains des rapports formés sont représentatifs de l'état nutritionnel d'une station, leur utilisation dans nos comparaisons de stations risque de faciliter grandement les problèmes d'échantillonnage. Il sera donc nécessaire de reprendre cette idée dans l'avenir.

\subsection{Liaisons entre les éléments et les caractères physiques autre que $\%$ de $\mathrm{H}_{2} \mathrm{O}$}

En début de saison, au moment du développement explosif des feuilles, les corrélations entre Ps et concentrations en éléments minéraux ont, en général, tendance à être négatives, sauf pour Ca (tableau 9). Cela traduit l'influence des effets de dilution à côté de celle des mécanismes d'ordre physiologique. Notons, cependant, que les liaisons Ps-concentrations s'estompent dans le courant de la saison de végétation : ainsi, l'influence des effets de dilution tendrait à diminuer en même temps que le taux de croissance des feuilles.

TABLEAU 9

1967 - Corrélations entre concentrations en éléments minéraux et "poids secs de 1000 feuilles $n$ ou Ps

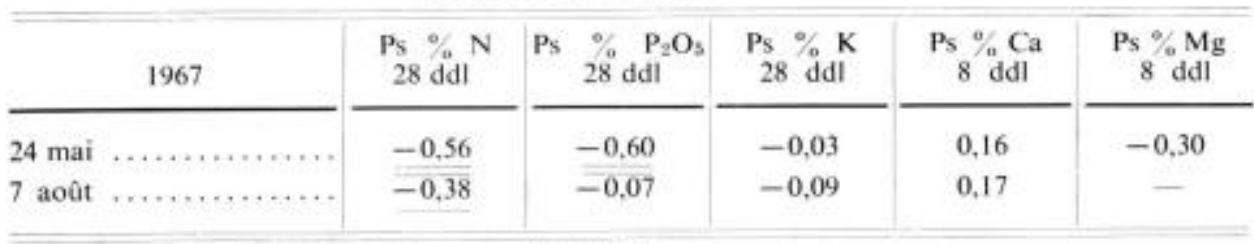

Corrélation négative au seuil de $1 \%$

Corrélation négative au seuil de $5 \%$ 
Dans un autre domaine, nous avons cherché à lier les concentrations en éléments minéraux aux dimensions individuelles des arbres. Comme il fallait s'y attendre, aucune liaison significative n'est apparue. Nous pourrions faire ici les mêmes remarques que celles que nous avions faites au sujet des pourcentages d'eau ou des Ps.

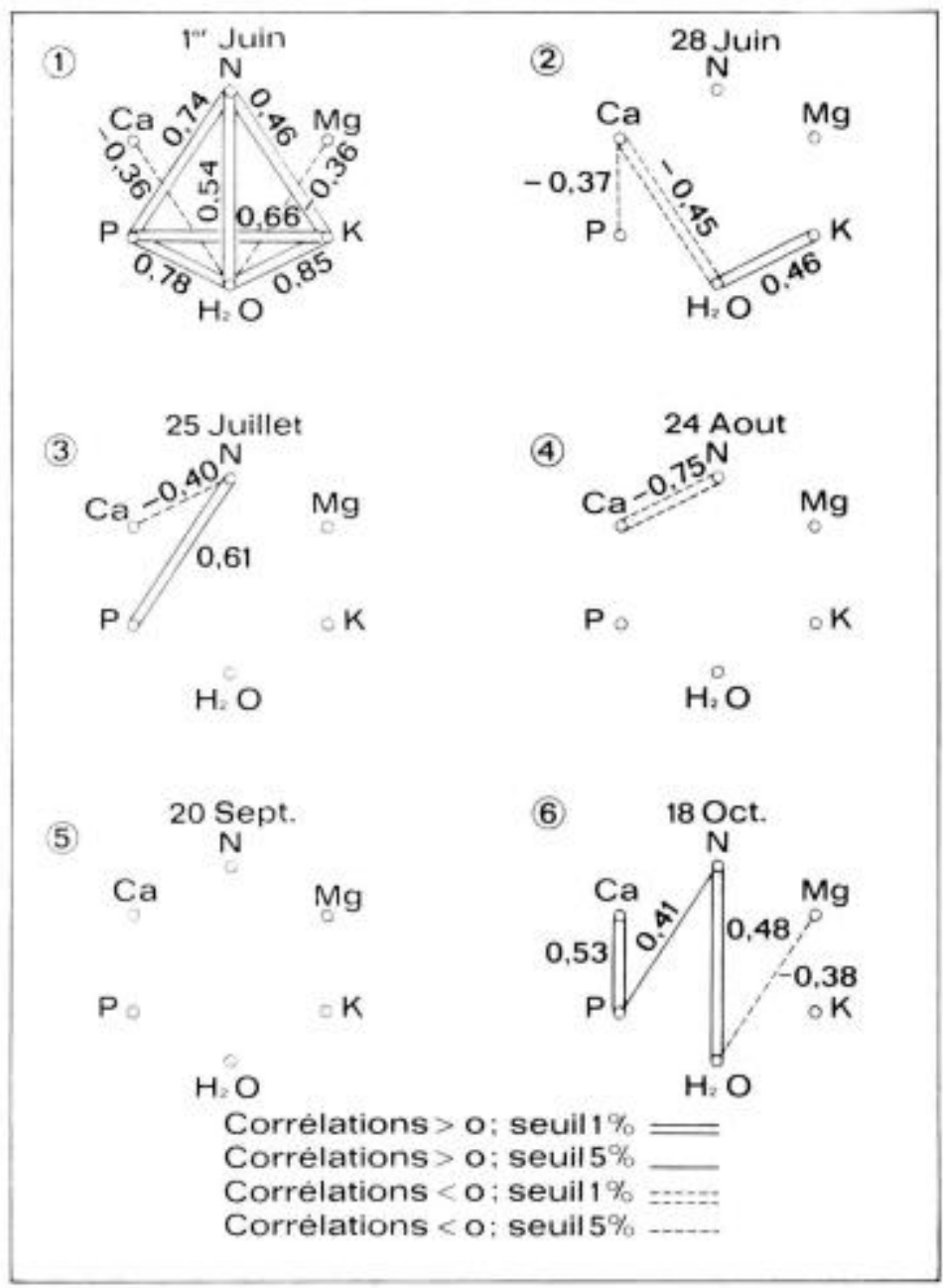

FiG, 8. - Corrélations teneurs en éléments minéraux et teneur en eau pour les 10 arbres pris individuellement et pour toutes les époques 1966 (27 d.d.l.).

Il semble cependant qu'en regroupant $\mathrm{Ps}, \% \mathrm{H}_{2} \mathrm{O}$ et $\%$ éléments nous commencerions à voir apparaître des liaisons entre les dimensions de l'arbre et cet ensemble de paramètres. Nous attendions des données plus précises pour envisager ces liaisons. 


\section{3. - Problèmes d'échantillonnage foliaire}

Le but premier de notre travail était de dégager les règles d'échantillonnage foliaire adaptées à notre programme de recherches sur le chêne. Les diverses remarques que nous avons pu faire contribueront en partie à l'élaboration de ces règles.

Nous allons successivement chercher à fixer la date, la position et le nombre d'arbres susceptibles de fournir les échantillons les plus représentatifs pour une étude de la nutrition minérale du chêne basée sur des comparaisons de stations.

\subsection{Epoque d'échantillonnage}

La simple observation des variations saisonnières moyennes de concentration en fonction du temps démontre la nécessité de déterminer avec soin l'époque à laquelle seront réalisés les prélèvements (fig. 3 à 7).

L'idéal serait d'opérer, par priorité, à une époque où les variations de concentration sont faibles. Malheureusement, ces époques différent pour chaque élément et même pour l'azote, il y a variation presque continue. D'autre part, un test de BARTLETT démontre que les dispersions des concentrations mesurées pour $\mathrm{P}, \mathrm{K}, \mathrm{Ca}$ et $\mathrm{Mg}$ différent significativement d'une date à l'autre. On s'aperçoit intuitivement de l'hétérogénéité des dispersions en mesurant sur les figures 4 à 7 la longueur des segments représentant l'intervalle de confiance de chaque moyenne. II s'avère donc nécessaire, en deuxième urgence, de choisir une date qui donnera globalement le minimum de dispersion pour l'ensemble des éléments.

Par ailleurs, nous n'en tiendrons pas compte pour le choix de l'époque des variations de concentration en $\mathrm{Ca}$ et $\mathrm{Mg}$. Nous verrons en effet, que le nombre d'arbres à échantillonner pour avoir une bonne précision sur ces éléments est si élevé qu'il serait illusoire de prendre ici ces éléments en considération

Enfin, l'époque devra également être choisie en fonction des remarques faites à propos des $\% \mathrm{H}_{2} \mathrm{O}$ et des $\mathrm{Ps}$.

En définitive, l'époque d'échantillonnage sera choisie essentiellement par rapport à $\mathrm{P}_{2} \mathrm{O}_{5}$ et $\mathrm{K}$. La période comprise entre les 15 et 30 août semble être la plus adaptée ou plutôt la moins mauvaise, puisqu'elle réunit un ensemble d'avantages et un minimum d'inconvénients qui apparaissent bien à la lecture des tableaux et figures.

Remarquons que nous avons fixé notre choix en fonction de notre programme de recherches. L'ensemble de nos résultats pourrait éventuellement concourir à la formulation de règles d'échantillonnage adaptées spécialement à des études de type différent.

En terminant, nous pouvons noter que les liaisons constatées entre éléments au début de saison ne sont pas intervenues dans le choix à l'époque d'échantillonnage. Ces liaisons peuvent traduire soit des rapports physiologiques spécifiques de l'espèce " chêne » soit un équilibre nutritionnel caractéristique de la station. Dans le cas où la seconde hypothèse se trouverait vérifiée par des travaux ultérieurs, il serait éventuellement intéressant de procéder aux échantillonnages juste après le 
débourrage. Bien sûr, dans ces conditions, les variations de concentration en fonction du temps seraient rapides ; toutefois, la connaissance des rapports inter-éléments serait peut-être suffisante pour définir l'état de nutrition d'une station.

\subsection{Position d'échantillonnage}

Rappelons que les variations de concentration en éléments pour chaque position en fonction du temps reproduisent assez fidélement les variations moyennes pour les trois positions. L'époque d'échantillonnage pouvait donc être choisie indépendamment de la position d'échantillonnage. Il s'agit maintenant de déterminer la position qui donnera une valeur à la fois précise et représentative des concentrations pour les éléments dosés.

Dans ce but, nous avons d'abord procédé à l'examen des variances des concentrations pour chaque élément et chaque niveau d'échantillonnage au 24 août 1966 : pour $\mathrm{N}, \mathrm{P}, \mathrm{K}$ et $\mathrm{Ca}$, la position médiane donne la variance minimum tandis que pour $\mathrm{Mg}$, ce minimum correspond à la partie supérieure de la cime. Toutefois pour $\mathrm{Mg}$, la variance calculée pour la position médiane est très proche de celle de la partie supérieure. Ainsi, à la lumière des résultats 1966, un échantillonnage dans la partie médiane de la couronne paraîtrait donner globalement la meilleure précision pour l'estimation des concentrations en $\mathrm{N}, \mathrm{P}, \mathrm{K}, \mathrm{Ca}$ et $\mathrm{Mg}$. Notons que pour les mesures et teneurs en eau et de poids secs, nous avions déjà retenu cette position médiane.

Nous pouvons d'autre part, vérifier (tableau 6) que cette position médiane donne également des valeurs des concentrations assez représentatives de l'ensemble du houppier. Pour $\mathrm{N}$ et $\mathrm{K}$, en effet, à une date donnée et particulièrement au mois d'août, la position d'échantillonnage intervient relativement peu sur les concentrations. Pour $\mathrm{P}_{2} \mathrm{O}_{5}$, Ca et $\mathrm{Mg}$, la position médiane paraît donner les concentrations les plus proches de la concentration « moyenne » $H M B$.

Il semble ainsi, après cette discussion, que la position médiane serait la plus adaptée pour nos échantillonnages.

\subsection{Nombre d'arbres composant l'échantillon}

Sur la base des résultats de la seule station de Champenoux, il est difficile de se faire une idée du nombre minimum d'arbres, susceptible de fournir un échantillon foliaire suffisant pour une étude par comparaison de stations. C'est, en effet, à partir des données correspondant aux diverses stations qu'on pourrait calculer ce nombre ; ce point sera donc abordé plus précisément à propos d'un travail en cours sur les stations de chêne.

Nous pouvons cependant essayer de donner, a priori, une réponse approximative grâce à la méthode statistique de DAvıEs, appliquée aux résultats de la station Champenoux (test à " deux queues » avec $\alpha=0,05$ et $\beta=0,2$ ). Ainsi, pour $\mathrm{N}, \mathrm{P}$ et $\mathrm{K}$, on peut affirmer qu'un écart de $15 \%$ sur la moyenne des concentrations est largement significatif quand l'échantillon groupe 10 arbres. Pour les $\% \mathrm{H}_{2} \mathrm{O}$, avec 10 arbres, un écart de $10 \%$ est significatif. Pour Ca et $\mathrm{Mg}$ par contre, il faudrait respectivement 25 à 45 arbres pour pouvoir affirmer que des écarts de $15 \%$ sont 
significatifs. Ces résultats à propos de $\mathrm{N}, \mathrm{P}, \mathrm{K}, \mathrm{Ca}$ et $\mathrm{Mg}$ coīncident d'ailleurs avec les chiffres proposés couramment pour les résineux.

Retenons donc pour l'instant, qu'un échantillon prélevé sur dix arbres risque de très bien convenir pour des comparaisons portant sur $\mathrm{N}, \mathrm{P}, \mathrm{K}$ ou le $\% \mathrm{H}_{2} \mathrm{O}$. En ce qui concerne Ca et $\mathrm{Mg}$, un tel échantillon a toutes chances de ne faire apparaitre que les écarts très importants. Pour les Ps, nous avions déjà précisé qu'avec dix arbres, on mettait en évidence des différences supérieures à $20 \%$. Terminons en soulignant qu'il est matériellement difficile d'envisager de prélever des échantillons sur plus de dix arbres.

\section{5. - CONCLUSIONS}

Les mensurations et analyses que nous avons pu réaliser jusqu'à maintenant sur une station de chêne en Lorraine, nous ont permis d'obtenir deux séries de résultats complémentaires. Les uns concernent des modalités d'échantillonnage ; les seconds éclairent un peu les phénomènes de distribution des éléments minéraux dans la masse foliaire et fournissent par ce biais des indications utilisables dans l'interprétation des analyses foliaires de chêne. En ce domaine, l'étude simultanée de plusieurs facteurs, poids et dimensions des feuilles, teneurs en eau, concentrations en éléments minéraux, s'est avérée très féconde.

Notre travail, original dans ses méthodes et dans le sens oủ les feuillus n'avaient guère attiré l'attention des chercheurs forestiers, nous a, en outre, conduit à soulever un certain nombre de questions qui fourniront matière à de nouvelles recherches.

Citons à ce propos nos remarques au sujet des liaisons et rapports interéléments ainsi que les questions relatives aux corrélations entre volume de l'arbre et certaines caractéristiques physiques ou chimiques de ses feuilles.

Nous avons rassemblé ci-dessous l'enssentiel de nos résultats :

- La croissance des feuilles est plus rapide en début qu'en fin de saison de végétation. Cependant, croissance en dimensions et augmentation de poids sec ne sont pas parallèles : tout se passe comme si les feuilles avaient tendance à acquérir leurs dimensions avant de fabriquer de la matière sèche. Sur une station riche, la croissance des feuilles de chêne pédonculé se caractérise par un taux relatif de croissance de l'ordre de $0,01 \mathrm{~g} / \mathrm{g} /$ jour pour l'ensemble de la saison de végétation.

- L'accroissement du poids sec des feuilles au cours de la saison s'accompagne d'une diminution de la teneur en eau de constitution (fig. 2).

- Du début à la fin de la saison de végétation, on observe une diminution des concentrations en $\mathrm{N}, \mathrm{P}, \mathrm{K}$ et $\mathrm{Mg}$ ainsi qu'une augmentation continue de la concentration en $\mathrm{Ca}$. Pour $\mathrm{N}$ et $\mathrm{Mg}$ la décroissance est continue alors que pour $\mathrm{P}$ et $\mathrm{K}$, un palier se manifestent en milieu de saison. Après le débourrage, les corrélations entre les concentrations des éléments pris deux à deux sont très fortes (fig. 3 à 7 ).

- Les dix arbres étudiés présentent entre eux des différences accentuées en ce qui concerne les poids secs de 1000 feuilles, les teneurs en eau et en éléments minéraux. Il semble possible d'attribuer ces caractères individuels à des facteurs 
essentiellement génétiques. Aucune corrélation n'est apparue entre le volume de l'arbre et chacun de ces caractères.

- La distribution des éléments minéraux dans le houppier est liée à la fois à des effets de dilution et à des mécanismes physiologiques. Toutefois, ces derniers semblent intervenir de façon prépondérante.

- Les phénomènes de transfert, ou translocation, des feuilles vers d'autres organes, apparaissent dès le milieu de la saison de végétation.

- Les feuilles du bas de la couronne donc globalement les feuilles d'ombre, sont plus grandes que les feuilles de lumière; elles présentent également des teneurs en eau et en éléments minéraux, sauf $\mathrm{K}$, plus fortes que les feuilles de lumière. Par contre, le poids sec de 1000 feuilles d'ombre est inférieur au poids sec de 1000 feuilles de lumière. Enfin, les feuilles de lumière maintiennent leur activité physiologique plus longtemps que les feuilles d'ombre.

- Le poids sec de 1000 feuilles et par là le poids d'éléments minéraux contenus dans 1000 feuilles sont beaucoup plus faibles en année sèche qu'en année humide.

- Pour une étude par comparaison de stations, on pouvait retenir les conditions d'échantillonnage suivantes :

— époque : $2^{e}$ moitié d'août

— position dans la couronne : tiers médian en prélevant tout autour de l'arbre

- nombre d'arbres à échantillonner : 10.

De cette façon, la précision sur N, P, K et les teneurs en eau est satisfaisante. Pour les poids secs de 1000 feuilles, Ca et $\mathrm{Mg}$, seules les différences importantes pourront être mises en évidence.

Terminons en rappelant que nos études de stations contribueront à éclaircir certaines de nos conclusions.

Reçu pour publication en mars 1968

\section{REMERCIEMENTS}

Nous remercions, C. MiLlifr, Station de Biométrie de I'INRA - Centre national de Recherches forestières, qui a bien voulu se charger de l'ensemble des calculs statistiques.

\section{SUMMARY}

SEASONAL CHANGES OF THE MAJOR ELEMENT COMPOSITION AND WATER CONTENT OF OAK-LEAVES (Quercux peduncalasa)

The purpose of the present study is to get some information on the foliar diagnosis on oak. These indications will allow the development of a research program on nutrition and production of oak by comparing stands. Later, this program will be extended to resinous, with hope to use oak as a site indicator of nutritive potentialities for resinous. We know, indeed, that oak is a widespread tree species in France. 
To obtain some indications on use of foliar diagnosis on oak, a stand of Qu. pedunculata has been selected on an eutrophic soil near Nancy. In that stand, ten dominant trees, twenty years old, have been chosen. The mean annual maximum increment amounts to $8.1 \mathrm{~m}^{3}$ per ha. Foliar samples have been collected on each of the ten trees, at three different crown elevations, during 1966 and 1967. The sample gathering was executed at regular intervals, between the beginning and the end of the growth season.

Every sample was submitted to a series of mensurations, weighings and analysis on N, P, K, $\mathrm{Ca}, \mathrm{Mg}$. So, it was possible to compare the variations of foliar ovendry weights and moisture contents with the changes of major element data. The essential of our results is gathered below :

a) The leaves tend to get their dimensions before making dry matter. The dry-weight increase of the leaves is accompanied by a decrease of moisture content (graph. 2).

b) During growth season, foliar contents in $\mathrm{N}, \mathrm{P}, \mathrm{K}, \mathrm{Mg}$ decrease while $\mathrm{Ca}$ content is increasing (graph. 3 à 7 ).

c) The ten trees present genetic differences for dryweights, moisture and mineral contents.

d) Physiologic mecanisms and dilution effects explain the distribution of minerals in the tree crown ; the first seem to have a preponderant action. Elsewhere, translocation effects appear from the middle of the growth season (Tab. 5).

e) The shade leaves are taller, richer in water and minerals, except $\mathrm{K}$, than the sun leaves. On the other hand, the dry weight of 1000 shade leaves is lower than the dry weight of 1000 sun leaves (Tab. 1, 2, 4, 5, 6 - Graph. 1).

f) For a comparative study of stands, samples have to be collected during the second half of August, in the middle third part of the crown and on ten trees. In this way, the accuracy is good for $\mathrm{N}, \mathrm{P}, \mathrm{K}$ and moisture contents. Important differences may appear only for $\mathrm{Mg}, \mathrm{Ca}$ and dry weights of 1000 leaves. We must observe that the present results would allow to elaborate different sampling rules adapted to a particular research programme.

Finally, it is interessing to note that the present study avises a big number of hypothesis and questions. Some of them will be studied again in our future works. The other belongs to pure physiology.

\section{ZUSAMMENFASSUNG}

DiE JAHREsZetTLICHEN SCHWANKUNGEN DES WASSER - UND NÄHRSTOFFGEHALTES IN DEN BLÄTtERN DER EICHE (Quercus pedunculata)

Die vorliegende Untersuchung hat zum Ziel, einige Hinweise beziuglich der Blattdiagnostik bei der Eiche zu erarbeiten. Diese Hinweise sollen die Erstellung eines Forschungsprogrammes ermöglichen, um Ernährung und Wuchsleistung der Eiche mit Hilfe von Standortsvergleichen zu untersuchen. Auf lange Sicht soll dieses Programm auch auf die Nadelhölzer erweitert werden, um bei Umwandlungen von Niederwald in Nadelholzbestānden, die Eiche als Indikator des potentiellen Năhrstoffgehaltes eines Standortes zu verwenden.

Die Eiche ist bekanntlich eine in Frankreich sehr weit verbreitete Holzart.

Um die Anwendungsmodalitäten der Blattdiagnostik bei der Eiche zu präzisieren, wurde in der Nähe von Nancy auf einem nährstoffreichen Pseudogleystandort ein 20 jähriger Eichenbestand (maximaler durchschnittlicher Zuwachs $8,1 \mathrm{~m}^{3}$ ) ausgewāhlt. An 10 herrschenden Bäumen wurden während der Vegetationsperioden der Jahre 1966 und 1967 in regeimāssigen Abstånden Blattstichproben in drei verschiedenen Höhen entnommen. An jeder einzelnen Stichprobe wurden eine Reihe von Messungen, Wägungen und Analysen $(\mathrm{N}, \mathrm{P}, \mathrm{K}, \mathrm{Ca}$ und $\mathrm{Mg}$ ) durchgeführt, sodass die Variationen von Trockengewicht, Wassergehalt und Nährstoffgehalt gleichzeitig verfolgt werden konnten.

Im folgenden werden die wesentlichen Ergebnisse kurz dargestellt :

a) Die Blätter zeigen die Tendenz, ihre Endgrösse vor Beginn einer nennenswerten Trocken. masseproduktion zu erreichen. Der Zuwachs an Trockenmasse ist mit einer gleichzeitigen Abnahme des Wassergehaltes verbunden (Abb, 2),

b) Wăhrend der Vegetationsperiode nehmen die Konzentrationen an $\mathrm{N}, \mathrm{P}, \mathrm{K}$ und $\mathrm{Mg} \mathrm{ab}$, während der Gehalt an $\mathrm{Ca}$ in den Blättern gleichmässig ansteigt. (Abb, 3 bis 7). 
c) Die 10 untersuchten Bāume sind untereinander bczüglich der Trockengewichte der 1000 Blätter sowie des Wasser-und Nährstoffgehaltes genetisch verschieden.

d) Die physiologischen Mechanismen und die Dilutionseffekte sind für die Verteilung der Nährstoffelemente in der Baumkrone verantwortlich ; die erstgenanten scheinen von grösserer Bedeutung, zu sein. Desweiteren konnten Translokationserscheinungen bereits in der Mitte der Vegetationsperiode beobachtet werden. (Tab, 5).

e) Die Schattenblätter sind grösser als die Sonnenblätter, sie haben einen höheren Wassergehalt und sind ( $\mathrm{K}$ ausgenommen) reicher an Nährstoffen. Im Gegensatz dazu, ist das Trockengewicht der 1000 Schattenblătter geringer (Tab, 1, 2, 4, 5, 6, Abb, 1).

f) Für eine Untersuchung von Standortsvergleichen wird eine Stichprobeentnahme in der zweiten Augusthälfte im mittleren Kronendrittel an 10 Bäumen vorgeschlagen. Man erhält auf diese Weise brauchbare Ergebnisse für N, P, K und den Wassergehalt ; man muss jedoch mit grösseren Differenzen bei $\mathrm{Ca}$ und $\mathrm{Mg}$ sowie bei den 1000 -Blattgewichten rechnen. Es wird jedoch besonders hervorgehoben, dass, auf Grund der vorliegenden Gesamtergebnisse, für besondere Fragestellungen entsprechende Stichprobeentnahmen ausgearbeitet werden können.

Abschliessend sei noch angeführt, dass die vorliegende Arbeit bestimmte Hypothesen und Fragen aufgeworfen hat, die, soweit sie nicht rein physiologischer Natur sind, in zukünftigen Arbeiten aufgegriffen werden sollen.

\section{RÉFÉRENCES BIBLIOGRAPHIQUES}

Cummings W.H.A., 1941. A method for sampling the foliage of silver maple tree. J. Forest., 39 , $382-386$.

Demolon A., 1956. Croissance des végétaux cultivés. Dunod éditeur Paris.

FIEDler H.J., HoHNE H., 1965. Vorkommen und Gehalt der Makronährstoffe in Waldbaümen. wissenschaftliche Zeitschrift der Technischen Universität - Dresden, I/4.

Grulimondi G, 1960. Ricerche preliminari sulla nutrizione minerale del pioppo a mezzo dell'analisi fogliare. Pubbl. centro sper, agric. for., 4, 231-245.

Guha M., Mitchell R.L.V., 1966. The trace and major element composition of the leaves of some deciduous trees. Seasonal changes. Plant and Soil, 24, 90-112.

Kozlowski T.T., 1966. Plant water relationships. The university of Wisconsin, U.I.R. Res. Newsletter, Nov. 66.

de LeENHEER L., 1963. L'analyse chimique du sol vue comme partic du grand problème de la fertilité du sol. Rev, Agric, belg., 10, oct, 63, 1513-1526.

MiLler W.F., 1966. Annual changes in foliar nitrogen, phosphorus and potassium levels of Lolbolly Pine with site and weather factors. Plant and Soil, 24, 369-378.

de Parcevaux S., 1963. Ed. INRA. Paris: L'eau et la production végétale, 63-150.

Qureshi I.M., Srivastava P.B.L., 1966. Foliar diagnosis and mineral nutrition of forest trees. The Indian Forester, 92-7, 1-18.

Van Der Meiden H.A., 1965, La fumure potassique du peuplier. Forẽt privée, nov. 64, janv. 65 , 123-127, 109-112.

WAREING P.F., 1966. The physiologist's approach to tree growth. Physiology in forestry, Supplement of forestry. Report of the sixth discussion meeting 1966, 7-18. 